Article

\title{
Are Superconductivity Mechanisms a Matter for Chemists?
}

\author{
Michel Pouchard * and Antoine Villesuzanne \\ CNRS, Université Bordeaux, Bordeaux INP, ICMCB, UMR 5026, F-33600 Pessac, France; \\ antoine.villesuzanne@icmcb.cnrs.fr \\ * Correspondence: michel.pouchard@icmcb.cnrs.fr
}

Received: 8 September 2020; Accepted: 22 October 2020; Published: 27 October 2020

check for updates

\begin{abstract}
From a tight-binding approach to the instability of nonbonding electronic states, along a double-well potential, we consider here how the coupling of these states with a phonon mode can open a superconducting gap at the Fermi level. The alternation of broken- and unbroken-symmetry states, along the phonon breathing distortion, induces the mixing of band-edge states on a very short timescale, according to the noncrossing rule of chemists. We show that this mixing may generate cationic and anionic disproportionation. The negative $U$ mechanism is thus justified here, leading to the mixing of occupied and unoccupied pair states, for the opening of a $2 \Delta$ superconducting gap. The closeness of broad $\sigma^{*}$ and narrow $\pi^{*}$ bands in the vicinity of the Fermi level should favor the superconducting phase over the insulating or metallic state, in agreement with Micnas et al.'s studies. We applied this approach to several families of superconducting materials, i.e., doped strontium titanate, high- $T_{\mathrm{C}}$ cuprates and iron selenide.
\end{abstract}

Keywords: nonbonding electrons; noncrossing rule; negative- $u$ mechanism; phonons; $\mathrm{BCS}$ theory; disproportionation; hole pairing; cuprates; titanates; iron selenide

\section{Introduction}

Are superconductivity mechanisms a matter for chemists? Such a question was a hot topic half a century ago, when the excitonic origin of superconductivity was widely discussed by scientists such as Schafroth, Blatt and Butler [1] or Little [2], in terms of strong interactions in real space ( $r$-space, familiar to chemists). However, the success of the Bardeen-Cooper-Schrieffer (BCS) theory [3], based on a delayed coupling between electrons of opposite spin and momentum, by exchange of phonons in reciprocal space (k-space), was immediately validated by experiment and discouraged many chemists from pursuing the r-space approach. Actually, the BCS theory predicted an upper value of ca. $30 \mathrm{~K}$ for the superconducting transition temperature $T_{C}$.

At its early stage, solid state chemistry rather focused on metal-insulator transitions, more spectacular as for example in vanadium oxides in which electric conductivity varies by more than ten orders of magnitude across the transition [4], and is correlated with small crystallographic changes (r-space). This is why many new families of solids, discovered by chemists, were only later found superconducting by physicists. In particular, this was the case of the so-called Chevrel phases discovered by Prigent, Sergent and Chevrel [5], for which superconductivity $\left(T_{C}=15 \mathrm{~K}\right)$ was evidenced two years later by Matthias [6].

A fourteen-year gap occurred between the synthesis of the first cuprates +III of lamellar perovskite type (Hagenmuller, Goodenough, Pouchard and Demazeau [7]) and the discovery of their superconductivity $\left(T_{C}=32 \mathrm{~K}\right)$ by Bednorz and Müller [8]; meanwhile, Raveau, Studer and Nguyen [9] had evidenced the key mixed valency +III/+II of copper in these systems. From then on, 
chemists participated actively in the so-called "golden rush" for new superconducting families and higher $T_{\mathrm{C}}$ values.

Thirty years later, there is still no consensus on the origin of this unconventional, high- $T_{\mathrm{C}}$ superconductivity: (bi-)polaronic, electronic, magnetic, excitonic, etc. Solving this mystery has been mostly the playground of physicists. However, a few chemists' concepts were proposed as early as 1987: copper + II disproportionation (similar to silver + II and gold + II) and oxygen to copper charge transfer [10-12], which have remained disregarded.

In 1990, Micnas, Ranninger and Robaczkiewicz [13] published a paper titled "superconductivity in narrow band systems with local non-retarded attractive interactions", which grabbed the attention of Simon concerning the properties of his rare-earth halogeno-carbides [14]. Actually, Micnas et al. provided an intermediate model, mixture of local pairs and itinerant electrons interacting via a charge-exchange mechanism, giving rise to a mutually induced superconductivity in these systems. Among popular topics in today's physics are the relations between three types of order, namely magnetism (spins), ferroelectricity (charges) and superconductivity (Cooper pairs); an example is multi-ferroicity. Solid state chemistry and its conceptual framework (structure-bonding-properties) are intrinsically involved in these three types of ordering.

In this article, we present a chemist's approach to the effect of doping in $\mathrm{SrTiO}_{3}$, which progressively turns from a quantum ferroelectric insulator to a metal and to a superconductor, as recently presented by Rischau et al. [15] and by Gabay and Triscone [16]. Then, while developing further some of our previous work [17], we describe the features underlying the transition from an antiferromagnetic Mott-type insulator such as $\mathrm{La}_{2} \mathrm{CuO}_{4}$ into a high- $T_{\mathrm{C}}$ superconductor. Finally, we tentatively apply these approaches to another family of high- $T_{C}$ superconductors, the iron pnictides/chalcogenides $[18,19]$.

\section{A Chemist's Scenario}

\subsection{Nonbonding Electrons, from Single to Double Well}

Cohesive energies in an ionocovalent solid can be described by a unique potential well, of Lennard-Jones form, with three terms arising from long-range Coulomb forces, short-range van der Waals (attractive) and Born forces (repulsive). As a function of the interatomic distance $d$, these three terms vary as $d^{-1}, d^{-6}$ and $d^{-12}$, respectively. A covalent bond, for which the potential well has a single minimum as a function of $d$, can be viewed as a charge accumulation in the interatomic region.

An atomic orbital (AO) basis set is often used to express the electronic structure, i.e., to expand molecular or crystal orbitals. From a molecular or local viewpoint, nonbonding electrons (NBEs) refer to molecular orbitals built on AOs with a vanishing overall overlap $S$. Overlap can be very small alike in $\delta$-type bonding (for example, $x y$-xy along the $\mathrm{z}$ direction) or vanish globally by symmetry, as shown hereafter in $\mathrm{CO}_{2}$.

\subsubsection{Point Symmetry and the $\mathrm{CO}_{2}$ Molecule}

For a $\mathrm{CO}_{2}$ molecule oriented along $z$, let us examine $\pi$-type molecular orbitals built on $2 \mathrm{p}_{\mathrm{x}} \mathrm{AOs}$ of carbon and oxygen atoms (labeled $\mathrm{O} 1$ and $\mathrm{O} 2$ ). A bonding, a nonbonding and an antibonding molecular orbital, respectively, noted as $1 \pi_{\mathrm{u}}, \pi_{\mathrm{g}}$ and $2 \pi_{\mathrm{u}}$, arise from the three $2 \mathrm{p}_{\mathrm{x}}$ AOs (Figure 1a). The nonbonding character of $\pi_{\mathrm{g}}$ results from the exact compensation of $2 \mathrm{p}_{\mathrm{x}}-2 \mathrm{p}_{\mathrm{x}}$ overlap integrals $S$, namely, $S(\mathrm{C}-\mathrm{O} 1)=-S(\mathrm{C}-\mathrm{O} 2)$ when the gerade combination of $\mathrm{O} 1$ and $\mathrm{O} 22 \mathrm{p}_{\mathrm{x}}$ orbitals is considered. If the symmetry is lowered by a displacement of the carbon atom with respect to the center of symmetry, as in a vibration mode, this molecular orbital will no longer be nonbonding, since the overlap integrals no longer compensate. The amplitude of the vibration mode depends on the shape of the potential well for the covalent bond, but also on the atom polarizability (related to Pearson's hardness in soft/hard chemistry concepts). 


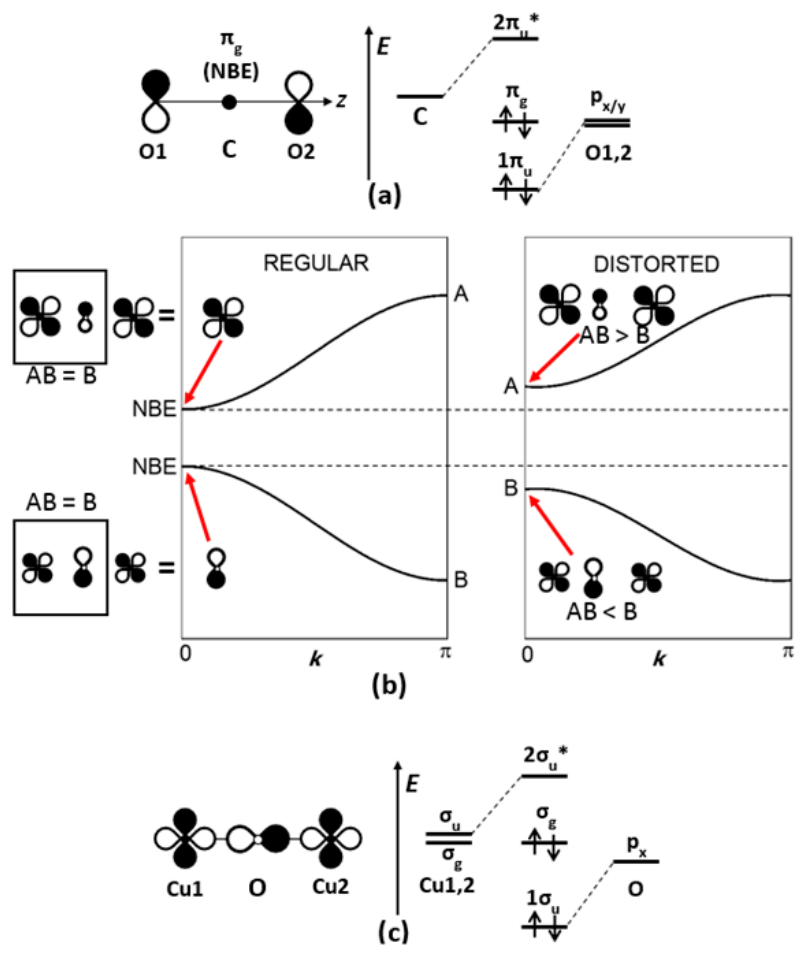

Figure 1. Nonbonding states. (a) By point-symmetry compensation: nonbonding $\pi_{\mathrm{g}}$ molecular orbitals of $\mathrm{CO}_{2}$. (b) By translational symmetry compensation: nonbonding crystal orbitals of a (TiO) $)_{\infty}$ chain. (c) Nonbonding spin-orbital $\sigma_{g}$ for a fragment $\mathrm{Cu}_{2} \mathrm{O}$ in a $(\mathrm{CuO})_{\infty}$ chain. Here NBE stands for nonbonding-electron states, while $\mathrm{B}$ and $\mathrm{AB}$ stand for bonding and antibonding, respectively.

\subsubsection{Translation Symmetry and Reciprocal Space}

The nonbonding character of a crystal orbital can arise from translational symmetry, for a given point or direction in the Brillouin zone. For a regular $(\mathrm{TiO})_{\infty}$ chain in $\mathrm{BaTiO}_{3}$ along the $c$ direction of the lattice, according to a tight-binding approach, some crystal orbitals are built on combinations of $\mathrm{O} 2 \mathrm{p}_{\mathrm{x}}$ and Ti $\mathrm{d}_{\mathrm{xz}}$ AOs. Among its quantum numbers, each crystal orbital $\varphi_{k}$ is characterized by a wave vector $k$ that belongs to the first Brillouin zone. If we restrict our example to a one-dimensional chain, then $k$ is a wave number with $-\pi / \mathrm{c}<k<\pi / \mathrm{c}$ and

$$
\varphi_{k}(r)=\sum_{n} e^{i k n c} \cdot\left\{c_{1}(k) \cdot \mathrm{d}_{\mathrm{xz}}(r)+c_{2}(k) \cdot 2 \mathrm{p}_{\mathrm{x}}(r)\right\}
$$

where the integer $\mathrm{n}$ indexes the unit cells, $c$ is the lattice parameter, $c_{1}$ and $c_{2}$ are normalization coefficients that depend on $k$. Figure $1 \mathrm{~b}$ shows a schematic energy diagram (or dispersion curves) $E\left(\varphi_{k}\right)$ vs. $k$ for the two levels or bands arising from the two AOs $2 \mathrm{p}_{\mathrm{x}}(\mathrm{O})$ and $\mathrm{d}_{\mathrm{xz}}(\mathrm{Ti})$. At the zone center $\Gamma(k=0), 2 p_{x}$ and $d_{x z}$ are not symmetry compatible and the crystal orbitals $\varphi_{k}$ are respectively of pure $2 p_{x}$ (top of valence band) and pure $d_{x z}$ (bottom of conduction band) character. The most bonding and antibonding combinations are found at the zone boundaries $(|k|=\pi / c)$. Therefore, the nonbonding states are found at the zone center, with energies of single $\mathrm{O} 2 \mathrm{p}_{\mathrm{x}}$ and Ti $\mathrm{d}_{\mathrm{xz}}$ AOs, respectively.

Any phonon mode displacing the oxygen atom along $c$, i.e., shortening vs. lengthening Ti-O bonds, allows bonding and antibonding Ti-O interactions to build up at the top of the valence band and at the bottom of the conduction band, respectively, at $\Gamma$. Consequently, the band gap increases and nonbonding states no longer exist at the band edges. In this case, the Lennard-Jones potential $E(q)$ centered at the equilibrium distance $(q=0)$ superimposes with an off-centered potential $E^{\prime}(q)$. The $q=0$ position is metastable for $\mathrm{E}(q)+E^{\prime}(q)$, which has a double-well shape. The double-well depth depends on the magnitude of short-range Born forces, oxygen ion polarizability and overlap/transfer integrals. 
According to the double-well depth, several behaviors arise: (i) the freezing of the phonon mode, into a ferroelectric-type distortion, (ii) an oscillation of the oxygen position, of the "transient crystal structure"-type, or (iii) the tunneling between the two potential wells of small depths. These behaviors were discussed recently by Gabay and Triscone as already cited [16], following a former description of the ferroelectric instability on the basis of chemical bonding, found in the textbook "Chemical Bonding in Solids" by Burdett and in a previous paper by Hoffmann et al. [20].

\subsubsection{Multielectron Systems: Hartree-Fock-Roothan Spinorbitals}

For a $\left[\mathrm{Cu}_{2} \mathrm{O}^{2+}\right.$ entity in a $(\mathrm{CuO})_{\infty}$ chain, two combinations of copper $\mathrm{x}^{2}-\mathrm{y}^{2}$ orbitals are possible, i.e., $\left(x^{2}-y^{2}\right)_{1} \pm\left(x^{2}-y^{2}\right)_{2}$, where indices 1 and 2 refer to the two copper atoms. The sum and difference of the $\mathrm{x}^{2}-\mathrm{y}^{2}$ orbitals have gerade $(\mathrm{g})$ and ungerade $(\mathrm{u})$ symmetry, respectively. Only the u combination allows a mixing with oxygen $2 p_{x}$ orbital, giving a bonding $\left(1 \sigma_{\mathrm{u}}\right)$ and an antibonding $\left(2 \sigma_{\mathrm{u}}{ }^{*}\right)$ molecular orbital (Figure 1c). Here, the oxygen $2 \mathrm{~s}$ semi-core orbitals were not considered (for further discussion, see Section 4.3.3). Then, the $g$ combination of $x^{2}-y^{2}$ orbitals results in a nonbonding molecular orbital (with respect to $\mathrm{Cu}-\mathrm{O}$ bonding); it is weakly $\mathrm{Cu}-\mathrm{Cu}$ bonding. This molecular orbital (noted $\sigma_{\mathrm{g}}$ hereafter) is expressed in the Hartree-Fock-Roothan formalism, using $x^{2}-y^{2}$ orbitals as basis sets. According to Deutscher and De Gennes [21],

$$
\begin{gathered}
\sigma_{g} \propto\left|\left(\mathrm{x}^{2}-\mathrm{y}^{2}\right)_{1} \overline{\left(\mathrm{x}^{2}-\mathrm{y}^{2}\right)_{2}}\right|+\left|\left(\mathrm{x}^{2}-\mathrm{y}^{2}\right)_{2} \overline{\left(\mathrm{x}^{2}-\mathrm{y}^{2}\right)_{1}}\right| \\
\quad+\left|\left(\mathrm{x}^{2}-\mathrm{y}^{2}\right)_{1} \overline{\left(\mathrm{x}^{2}-\mathrm{y}^{2}\right)_{1}}\right|+\left|\left(\mathrm{x}^{2}-\mathrm{y}^{2}\right)_{2} \overline{\left(\mathrm{x}^{2}-\mathrm{y}^{2}\right)_{2}}\right|
\end{gathered}
$$

The first two terms are of covalent character and related to the antiferromagnetic coupling between copper sites (Goodenough-Kanamori type). The last two terms are of ionic character and correspond to a $2 \mathrm{Cu}^{2+} \rightarrow \mathrm{Cu}^{+}+\mathrm{Cu}^{3+}$ disproportionation, which was proposed in 1987 as a possible origin of Cooper pairs in cuprates (as mentioned in the introduction) [10-12], and which is closely related to the Hubbard parameter $U$ for electron-electron repulsion. Those ionic terms indicate that, similarly to $\mathrm{BaTiO}_{3}$, a succession of disproportionate states may occur, making the mid position of the $\mathrm{Cu}-\mathrm{O}-\mathrm{Cu}$ bridge metastable for oxygen; due to the difference in the ionic radii of $\mathrm{Cu}^{+}$and $\mathrm{Cu}^{3+}$, the oxygen atom should move away from the doubly occupied single-site orbital $\left(\mathrm{Cu}^{+}\right)$. As in $\mathrm{CO}_{2}$, this symmetry breaking allows the oxygen $2 \mathrm{p}_{\mathrm{x}}$ orbital to mix with the $\mathrm{x}^{2}-\mathrm{y}^{2}$ orbitals in $\sigma_{\mathrm{g}}$, mostly on the $\mathrm{Cu}^{3+}$ site. Phonons will further amplify this symmetry-broken situation, to possibly achieve a permanent distortion at low temperature. Furthermore, cuprates can host other instabilities in which phonons are key parameters, such as Fermi surface nesting and orbital ordering.

From this chemical approach, we conclude that nonbonding electron (NBE) states are very unstable with respect to symmetry-breaking perturbations such as phonons. The electronic structure instantaneously adapts to the atomic oscillations to minimize the energy of the system; as quoted by Maupertuis three centuries ago: "Nature always uses the simplest means to accomplish its effects". The key question here is the time scale: atomic oscillations occur at the picosecond scale, while electrons visit interatomic distances at the tens of attosecond scale only. Down to which time scale can we talk about succession of states, instead of state mixing?

\subsection{Sketching a Chemical Scenario}

We emphasized the instability of an NBE state toward static or dynamical distortions in high-symmetry sites. A phonon mode can induce a succession of symmetry-broken and symmetry-unbroken states, with inverse local distortions at each half-period. Obviously, symmetry-broken states last much longer than intermediate high-symmetry states, along one period of vibration. Even if formally instantaneous, we will consider a very short time span $\delta t$ for the symmetry-unbroken state, with respect to the period.

The period increases along with the softening of the phonon mode; for narrow bands (in under-doped samples), the phonon softening with decreasing temperature leads eventually 
to an ordered phase, of short or long range, with the freezing of charge transfer, magnetic or orbital order, and stripe or checkerboard-type structures. For broad bands (in over-doped samples), and beside the very short $\delta t$ time span, the softening should lead to a metallic phase of the Fermi-liquid type. For intermediate band widths, the symmetry breaking induces a gap between the occupied electron band and the unoccupied band. A half-period later, the role of the two bands reverses along with a band crossing. The noncrossing rule (NCR), shown in Figure 2 for discrete states and for crystal orbital states [22], can be retrieved if a superconducting (BCS type) gap opens, which involves the mixing of occupied $\left(\varphi_{\mathrm{o}}\right)$ and unoccupied $\left(\varphi_{\mathrm{u}}\right)$ pair states, of wave vectors $(\boldsymbol{k},-\boldsymbol{k})$ and $\left(\boldsymbol{k}^{\prime},-\boldsymbol{k}^{\prime}\right)$ respectively, according to Whangbo [23]:

$$
\left\langle\Phi_{\mathrm{o}}(\boldsymbol{k}) \cdot \Phi_{\mathrm{o}}(-\boldsymbol{k})\left|H^{\prime}\right| \Phi_{\mathrm{u}}\left(\boldsymbol{k}^{\prime}\right) \cdot \Phi_{\mathrm{u}}\left(-\boldsymbol{k}^{\prime}\right)\right\rangle
$$
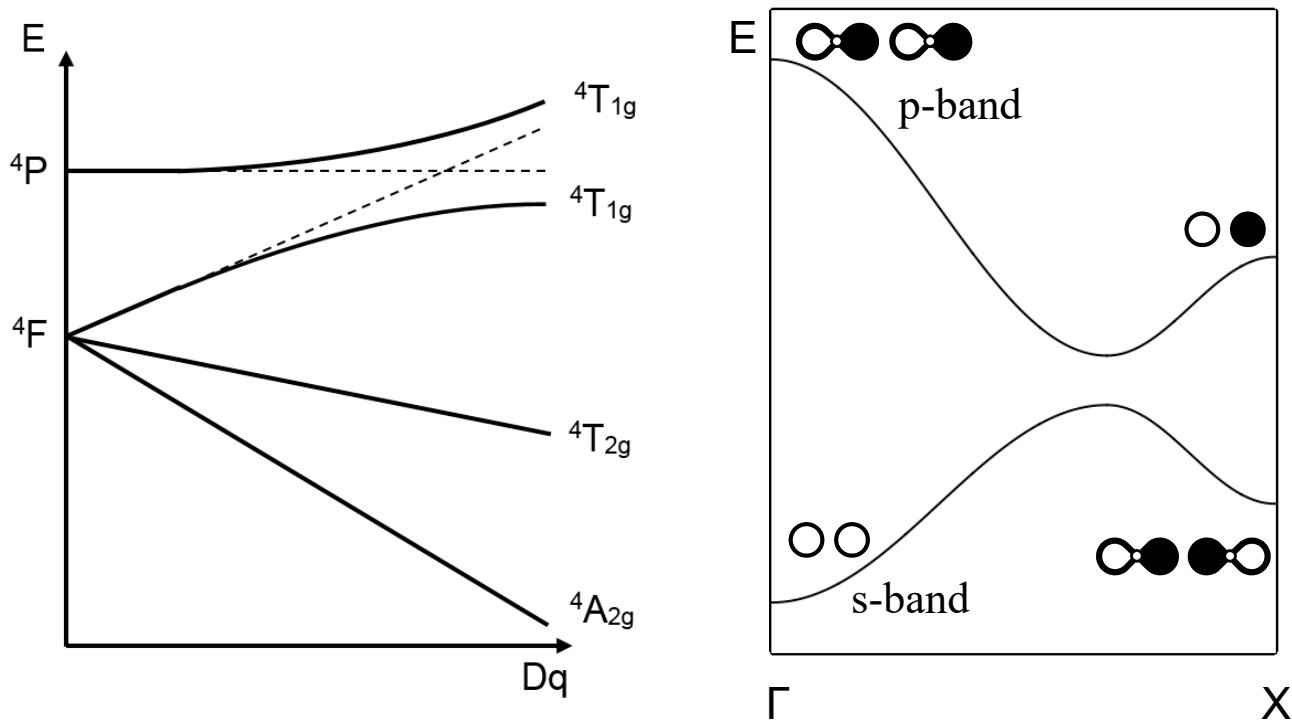

Figure 2. Schematic representations of the NCR for state ${ }^{4} \mathrm{~T}_{1 \mathrm{~g}}\left({ }^{4} \mathrm{~F},{ }^{4} \mathrm{P}\right)$ of $\mathrm{Cr}^{3+}[22]$, and for a $1 \mathrm{D}$ band with s-p hybridization.

The nucleation of the superconducting phase will be favored if pseudo-local pair states are also present at the Fermi level, i.e., if the bands above cross a non-dispersive band such as those arising from carbon atomic clusters in rare earth halogeno-carbides (for example in $\mathrm{Y}_{2} \mathrm{I}_{2} \mathrm{C}_{2}$ with $T_{\mathrm{C}}=10 \mathrm{~K}$ [14]).

\section{Metallic and Superconducting Behavior of $\mathrm{SrTiO}_{3}$}

From the crystallographic point of view, $\mathrm{SrTiO}_{3}$ is a perfect perovskite; its Goldschmidt factor is strictly equal to unity. When stoichiometric, $\mathrm{SrTiO}_{3}$ is a dielectric insulator with the relative permittivity rising to about 20,000 at low temperature, typical of a quantum ferroelectric. The substitution of $\mathrm{Ca}^{2+}$ for $\mathrm{Sr}^{2+}$ (a few \%), with different ionic radii, induces a ferroelectric instability. On the other hand, a slight non-stoichiometry due to oxygen vacancies generates n-type charge carriers and a metallic state, and even a superconducting state below $1 \mathrm{~K}$, despite a very small carrier density $[15,24]$. Soft phonon modes and oxygen non-linear polarizability were proven to play a key role in the ferroelectric and superconducting properties of $\mathrm{SrTiO}_{3}$, among other systems [25-27]. As mentioned earlier, there might exist a link between the charge ordering in an insulating ferroelectric phase and the spin/momentum ordering in a superconducting phase. Actually, materials both metallic and polar are scarce and poorly understood so far [16]. In 2017, Rischau et al. proposed that these two kinds of order can coexist upon homogeneous doping, even reinforce each other [15]; however, many works have evidenced inhomogeneous doping in $\mathrm{SrTiO}_{3}$ as well, resulting in metallic regions coexisting with insulating ones [28-30]. 
Is there a chemical explanation to those unconventional results? In the first part, we showed how the nonbonding electrons of pure oxygen character, at the top of the valence band, could become partly bonding following a tetragonal distortion of ferroelectric type in $\mathrm{BaTiO}_{3}$. Conversely, the nonbonding states of pure Ti-character are destabilized; the band gap increases, but the stability of the compound is not affected since the conduction band is empty. The latter argument is no longer valid if electrons are introduced by doping: their destabilization competes with the stabilization of the top of the valence band, and thus with the ferroelectric instability itself. This is observed in tungsten bronzes such as $\mathrm{Na}_{x} \mathrm{WO}_{3}(0<\mathrm{x}<1)$, known for more than a century, in which the ferroelectric instability of $\mathrm{WO}_{3}$ disappears progressively for $\mathrm{x}$ up to ca. 0.2 [31]. It is therefore understandable that superconductivity in $\mathrm{SrTiO}_{3}$ remains limited by the extent of electron doping $(<0.001)$. Two preliminary remarks must be made:

(i) The Mott-type insulator-metal transition limits the value of $a_{H}^{*} \sqrt[3]{n}$ close to 0.2 , where $n$ is the carrier density and $a_{H}^{*}$ is the Bohr radius corrected by the dielectric constant, as illustrated by Edwards and Sienko [32]. When the dielectric constant is very high, $a_{H}^{*}$ may reach values as high as a micrometer; this is precisely what occurs in $\mathrm{SrTiO}_{3}$ at low temperature.

(ii) The ferroelectric distortion induces a splitting of the conduction band, namely, $\pi^{*}\left(\mathrm{e}_{\mathrm{g}}\right)$ and $\pi^{*}\left(\mathrm{~b}_{2 \mathrm{~g}}\right)$, where $e_{g}=(x z, y z)$ and $b_{2 g}=x y$ (Figure 3$)$.

\section{$\mathrm{C}_{4 \mathrm{v}}$ crystal field splitting}
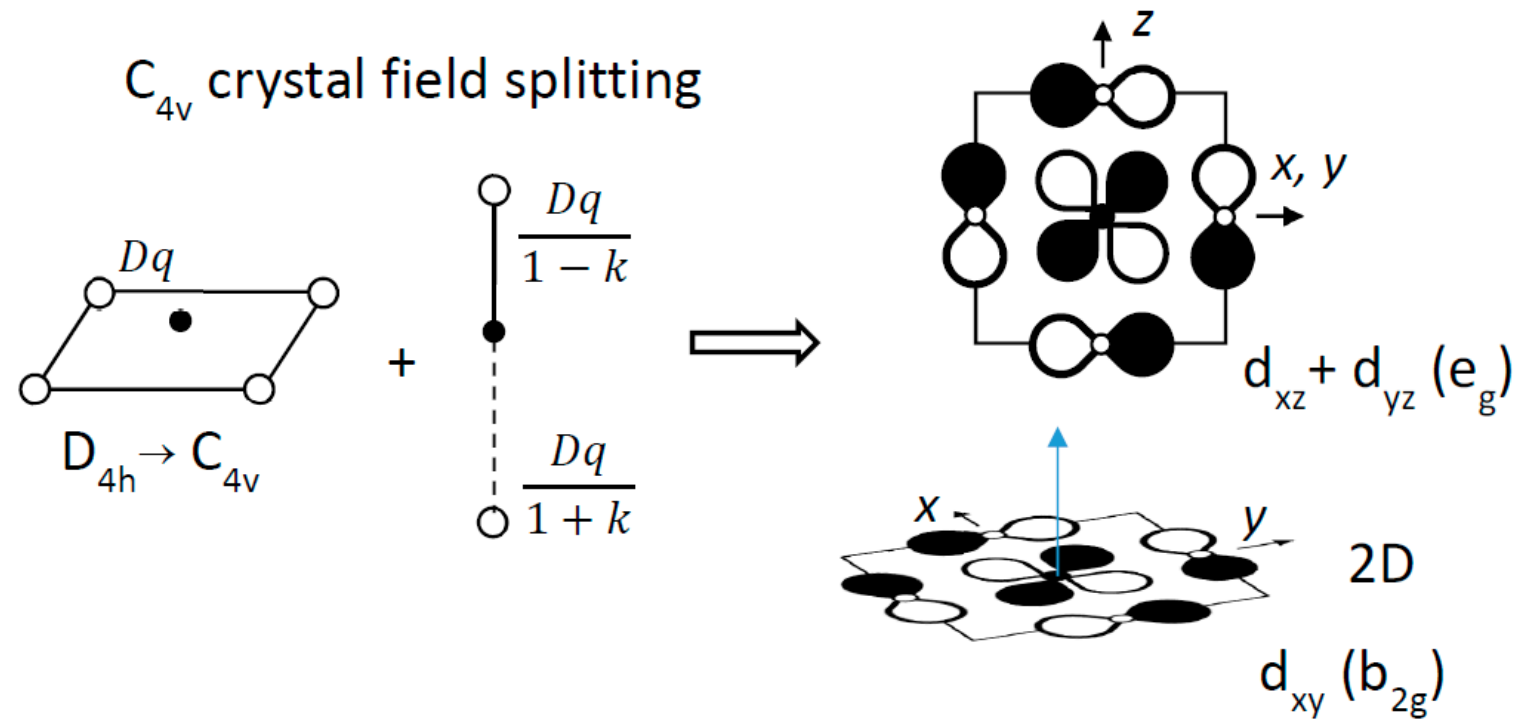

Figure 3. Crystal field splitting of $t_{2 g}$ atomic orbitals from $\mathrm{O}_{h}$ to $\mathrm{C}_{4 \mathrm{v}}$ symmetry, from the ligand field contributions of two axial ligands, unsymmetrically coordinated to the metal ion.

A simple crystal field consideration accounts qualitatively for this band splitting, by summing the potentials in units of $D q$, the phenomenological parameter used by chemists. The short (axial), the long (axial), and the normal (equatorial) Ti-O interactions are described by the potentials $\mathrm{Dq} /(1-\mathrm{k})$, $\mathrm{Dq} /(1+\mathrm{k})$ and $\mathrm{Dq}$, respectively $(0<\mathrm{k}<1)$. The band splitting leads to a stabilization of the $\mathrm{b}_{2 \mathrm{~g}}$ state $\left(-6.28 \mathrm{k}^{2}\right)$ with respect to the $\mathrm{e}_{\mathrm{g}}$ state $\left(+1.14 \mathrm{k}^{2}\right)$. From the point of view of chemical bonding, the $\mathrm{Ti}^{4+}$ displacement above (or below) the $\mathrm{O}_{4}$ plane (i) lifts the degeneracy of $\mathrm{t}_{2 \mathrm{~g}}$ states and (ii) decreases significantly the $3 \mathrm{~d}_{\mathrm{xy}} / 2_{\mathrm{p}}$ overlap, decreasing consequently the width of the $\mathrm{b}_{2 \mathrm{~g}}$ band (as well known for the $\mathrm{VO}^{2+}$ vanadyl ion).

The first carriers then occupy the narrow $2 \mathrm{D} \pi^{*}\left(\mathrm{~b}_{2 \mathrm{~g}}\right)$ band, of the fermion character, but also as electron pairs of the boson type, since their Coulomb repulsion on the same orbital almost vanishes $(U \approx 0)$. Their coherence length should be close to the one in BCS-type superconductors such as $\mathrm{Nb}_{3} \mathrm{Sn}$ (few hundreds $\mathrm{nm}$ ). Under the phonon modes discussed above, those pseudo-local pairs, which are not yet proper Cooper pairs, mix, at each $\frac{1}{4}$ period, with empty $t_{2 g}$ states arising from $t_{2 g}=b_{2 g}+e_{g}$. 
Then, they can separate according to $\mathrm{b}_{2 \mathrm{~g}}{ }^{2} \rightarrow \mathrm{b}_{2 \mathrm{~g}}{ }^{1}+\mathrm{e}_{\mathrm{g}}{ }^{1}$ (" $\mathrm{t}_{2 \mathrm{~g}}{ }^{2 \text { ") }}$ into a Fermi liquid, or mix with empty $e_{g}$ or $t_{2 g}$ states according to $b_{2 g}{ }^{2}$ (pseudo-local pairs) $+t_{2 g}{ }^{0}\left(" t_{2 g}{ }^{l t \prime}\right)$ into Cooper pairs with opening of a superconducting gap.

$$
\left\langle\mathrm{b}_{2 \mathrm{~g}}^{1}(k) \cdot \overline{\mathrm{b}}_{2 \mathrm{~g}}^{1}(-k)\left|H^{\prime}\right| \mathrm{t}_{2 \mathrm{~g}}^{0}\left(k^{\prime}\right) \cdot \overline{\mathrm{t}}_{2 \mathrm{~g}}^{0}\left(-k^{\prime}\right)\right\rangle
$$

In essence, the ferroelectric instability (provided by the small doping of $\mathrm{Ca}^{2+}$ and by the nonbonding character of electrons at the top of the valence band) induces a bistable electron system coupled to the oscillations of the axial oxygen atom. Here, we had all the ingredients for a two-band scenario, with a broad and a narrow band; the first band consists of empty states, the second contains electron pairs due to $U \approx 0$ and a large dielectric constant. The two bands mix before the gap closure, into a superconducting state even with such low carrier densities as $10^{17} \mathrm{~cm}^{-3}$.

A simple electronic Hamiltonian $\mathrm{H}^{\prime}$ replaces here the (delayed) electron-phonon coupling Hamiltonian of the BCS model. This chemist's view applies to $\mathrm{SrTiO}_{3}-\mathrm{LaAlO}_{3}$ heterostructures as well, justifying the intrinsic doping and the ferroelectric-type distortions expected in these structures. $\mathrm{SrTiO}_{3}$ can be viewed as a succession of neutral $\mathrm{TiO}_{2}$ and $\mathrm{SrO}$ layers; $\mathrm{LaAlO}_{3}$, a perovskite as well, has charged $\mathrm{AlO}_{2}{ }^{-}$and $\mathrm{LaO}^{+}$layers. Thus, the interface of the heterostructures can be regarded as a charged layer $\mathrm{La}_{0.5} \mathrm{Sr}_{0.5} \mathrm{O}^{+0.5}$. The electric field gradient between $\mathrm{LaO}^{+}$and neutral $\mathrm{SrO}$ layers should be screened, as in p- $\mathrm{n}$ junctions, by a charge transfer: $\mathrm{TiO}_{2}+\mathrm{AlO}_{2}{ }^{-} \rightarrow\left(\mathrm{TiO}_{2}\right)^{-\delta}+\left(\mathrm{AlO}_{2}\right)^{-(1-\delta)}$, which is actually a charge transfer between $\mathrm{O}^{2-}\left(\mathrm{AlO}_{2}\right)$ and $\mathrm{Ti}^{4+}\left(\mathrm{TiO}_{2}\right)$; the $\mathrm{e}^{-} / \mathrm{h}^{+}$pair opposes then the electric field gradient.

Additionally, the Ti-O-Al entity is dissymmetrical in terms of chemical bonding and, in the same way as a ferroelectric distortion, induces both a band splitting from $t_{2 g}$ to $b_{2 g}+e_{g}$ and a dynamical polarization of $\mathrm{TiO}_{2}$ layers away from the interface. Therefore, the first nonbonding electrons in the conduction band $\left(t_{2 g}\right)$ will undergo a succession of quasi-local pair states $\left(b_{2 g}{ }^{\natural}\right.$, few hundreds $\left.n m\right)$ and delocalized Cooper-pair states, due to the mixing with empty $e_{g}$ or $t_{2 g}$ states when the gap closes. Such a description is close to that of Gariglio et al. [33] in their review on interface superconductivity.

\section{High- $T_{\mathrm{C}}$ Superconducting Cuprates}

A significant difference between cuprates and titanates is the one-electron energy (Coulomb integral) for $3 \mathrm{~d}$ orbitals. The $\mathrm{Ti}$ and $\mathrm{Cu} 3 \mathrm{~d}$ states lie respectively above and below the $\mathrm{O} 2 \mathrm{p}$ state, which affects the electronic structure: in cuprates with optimum doping, the valence band should be of dominant $\mathrm{Cu}$-character, the conduction band being mostly of oxygen character.

$\mathrm{La}_{2} \mathrm{CuO}_{4}$, the structural parent of superconducting cuprates, is a charge transfer insulator with a first excited state described by $\mathrm{O}^{2-}+\mathrm{Cu}^{2+} \rightarrow \mathrm{O}^{-}+\mathrm{Cu}^{+}$. It is also an antiferromagnet with a Néel temperature close to the ambient temperature. $\mathrm{La}_{2} \mathrm{CuO}_{4}$ is mostly p-doped by substitution of $\mathrm{Ba}^{2+}$ or $\mathrm{Sr}^{2+}$ for $\mathrm{La}^{3+}$, or by chemical or electrochemical intercalation of oxygen in $\mathrm{La}_{2} \mathrm{O}_{2}$ rock salt-type layers. Doping of $8-20 \%$ induces a metallic then superconducting state $\left(T_{C} \approx 40 \mathrm{~K}\right)$, along with a significant decrease in the unit cell volume; shortened interatomic distances lead to larger $\mathrm{t}_{\mathrm{Cu}-\mathrm{O}}$ transfer integrals and thus to larger band widths, but also to stronger oxygen-oxygen interactions that turn out to be of major relevance here. Along with doping, the band widths increase and the value of the Hubbard parameter decreases due to covalency ( $U$ is related to Racah repulsion parameters $B$ and $C$, familiar to chemists, and sensitive to covalency); this is consistent with an insulator-metal transition of the Mott-Hubbard type, for increasing the $t / U$ ratio.

We start from this framework, with $U \approx 0$, to describe the electronic structure of cuprates with optimum doping (i.e., formally $\mathrm{Cu}^{(2+x)+}$ with $x$ close to 0.15 ). $U \approx 0$ means a full $\mathrm{O}^{2-}+\mathrm{Cu}^{2+} \rightarrow \mathrm{O}^{-}+\mathrm{Cu}^{+}$ transfer, $\mathrm{Cu}^{+} / \mathrm{O}^{-}$being the ground state. This is justified by several arguments:

(i) Coulomb integrals, $H_{\mathrm{dd}}(\mathrm{Cu})=-18.7 \mathrm{eV}$ and $H_{\mathrm{pp}}(\mathrm{O})=-17.1 \mathrm{eV}$ [34]. Tight-binding calculations for $\left(\mathrm{CuO}_{4}\right)^{5-}$ square planar clusters, as found in $\mathrm{La}_{2} \mathrm{Li}_{0.5} \mathrm{Cu}_{0.5} \mathrm{O}_{4}$ [35], show clearly this charge transfer situation (Figure 4 in Ref. [36]). 
(ii) Doped holes in cuprates are essentially located on oxygen atoms, as demonstrated by the pioneering work of Bianconi et al. [37] by X-ray absorption spectroscopy (XANES), later on by electron-loss spectroscopy (EELS) [38] and by spectacular atomic scale imaging from tunneling asymmetry, even on underdoped samples, by Hanaguri et al. [39], Kohsaka et al. [40] and Pasupathy et al. [41], showing inhomogeneous charge distributions as nano-scale stripes or checkerboard patterns on oxygen atoms.

(iii) Recent studies by Jurkutat et al. [42] using NMR on ${ }^{63-65} \mathrm{Cu}$ and ${ }^{17} \mathrm{O}$ doped samples, leading to a phase diagram for $T_{\mathrm{C}}$ as a function of the distribution of doped holes at $\mathrm{Cu}$ and $\mathrm{O}$ sites.

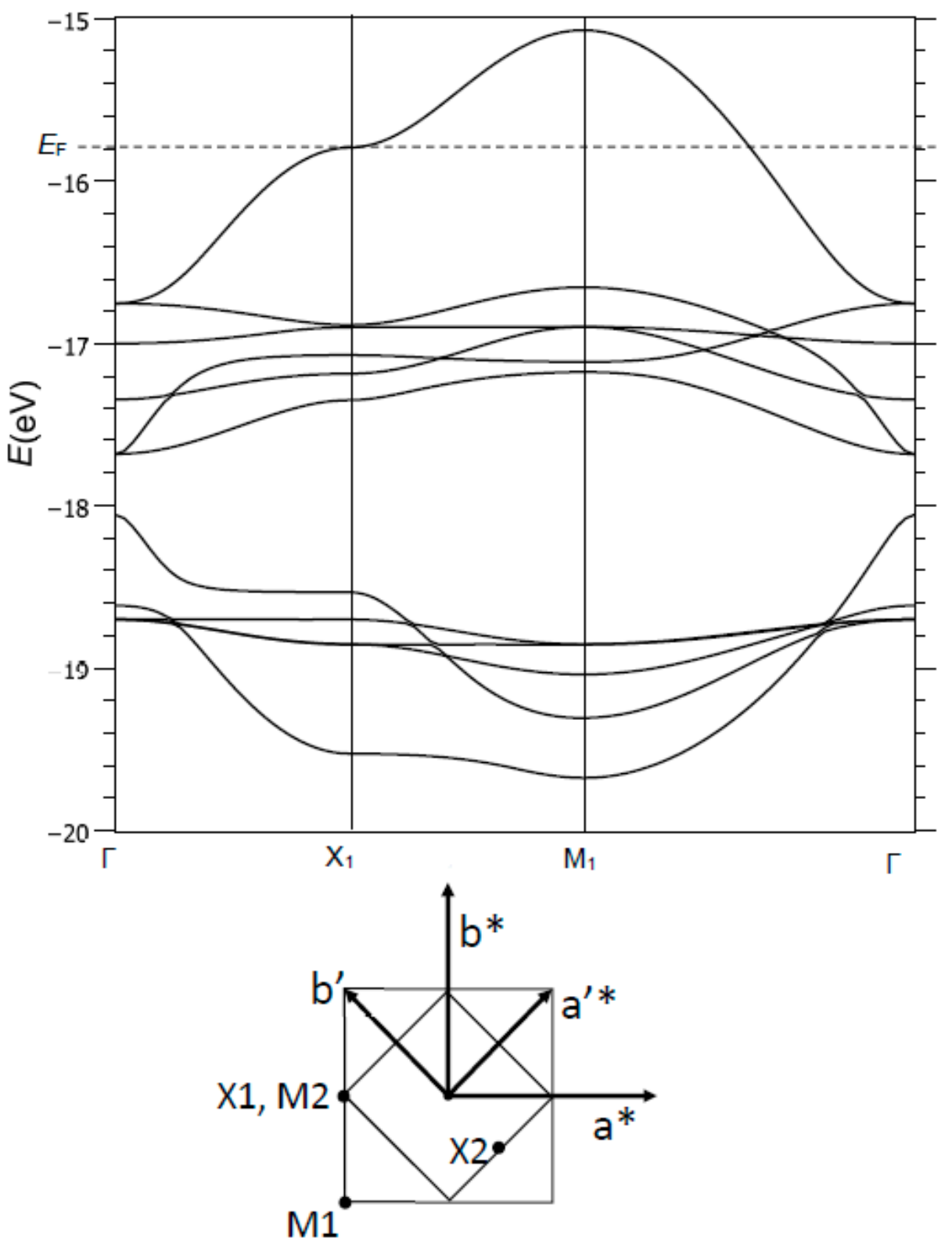

Figure 4. Top: Dispersion curves $E(k)$ for a $\left(\mathrm{CuO}_{2}\right)^{2-}$ unit cell, from tight-binding calculations. Bottom: Brillouin zones for the single and double unit cells (BZ1 and BZ2), and their high symmetry points.

\section{1. $\left(\mathrm{CuO}_{2}\right)^{2-}$ Unit Cell}

The input data for our tight-binding calculations are reported in Table 1. Figure 4 (top) shows the dispersion curves $E(k)$ in the -19 to $-15 \mathrm{eV}$ range. Several bands are identified:

(i) Around $-19 \mathrm{eV}$, a valence band of $\mathrm{Cu}$ character and $\mathrm{Cu}-\mathrm{O}$ bonding interactions, 
(ii) Between -18 and $-15 \mathrm{eV}$, a broad conduction band of oxygen character, and $\mathrm{Cu}-\mathrm{O}$ generally antibonding.

Table 1. Parameters for extended Hückel tight-binding calculations: $H_{\mathrm{ii}}{ }^{\prime} \mathrm{s}$ are the diagonal matrix elements of the effective Hamiltonian, $\zeta_{i}$ 's and $c_{i}$ 's are respectively the exponents and contraction coefficients for the Slater-type atomic orbitals (AOs).

\begin{tabular}{ccccccc}
\hline Element & AO & $\boldsymbol{H}_{\text {ii }}(\mathbf{e V})$ & $\zeta_{\mathbf{1}}$ & $\mathbf{c}_{\mathbf{1}}$ & $\zeta_{\mathbf{2}}$ & $\mathbf{c}_{\mathbf{2}}$ \\
\hline $\mathrm{Cu}$ & $4 \mathrm{~s}$ & -7.44 & 2.151 & 0.48071 & 1.168 & 0.61474 \\
& $4 \mathrm{p}$ & -4.26 & 1.370 & 1.0 & & \\
$\mathrm{O}$ & $3 \mathrm{~d}$ & -18.7 & 7.025 & 0.44221 & 3.004 & 0.68886 \\
& $2 \mathrm{~s}$ & -33.7 & 2.246 & 1.0 & & \\
$\mathrm{Fe}$ & $2 \mathrm{p}$ & -17.1 & 2.227 & 1.0 & & \\
& $4 \mathrm{~s}$ & -9.1 & 1.900 & 1.0 & & \\
& $4 \mathrm{p}$ & -5.32 & 1.900 & 1.0 & & \\
$\mathrm{Se}$ & $3 \mathrm{~d}$ & -12.6 & 5.350 & 0.55050 & 2.000 & 0.62600 \\
& $4 \mathrm{~s}$ & -20.5 & 2.440 & 1.0 & & \\
& $4 \mathrm{p}$ & -13.2 & 2.070 & 1.0 & & \\
\hline
\end{tabular}

Figure 5 shows an enlarged view of the two top bands, between -17 and $-15 \mathrm{eV}$, noted here $\sigma^{*}\left(b_{1 g}\right)$ and $\pi^{*}\left(a_{2 g}\right)$ (as referred to the point symmetry of the copper site, at the M1 point of BZ1). At $\Gamma$, these two bands are degenerate and of pure oxygen character (Figure 5a). The top band, $\sigma^{*}\left(b_{1 g}\right)$, is broad $(W \approx 1.5 \mathrm{eV})$ due to strong $\mathrm{Cu}-\mathrm{O}$ and $\mathrm{O}-\mathrm{O}$ antibonding interactions; the $\pi^{*}\left(\mathrm{a}_{2 \mathrm{~g}}\right)$ band is narrow $(W \approx 0.3 \mathrm{eV})$ because of its $\mathrm{Cu}-\mathrm{O}$ nonbonding character (especially in $\Gamma, \mathrm{M} 1$ ) or by compensation of $\mathrm{Cu}-\mathrm{O}$ and $\mathrm{O}-\mathrm{O}$ antibonding/bonding interactions (in $\mathrm{X} 1$ ).

The $E(k)$ vs. $k$ plot for $\sigma^{*}\left(\mathrm{~b}_{1 \mathrm{~g}}\right)$ shows a saddle point in $\mathrm{X} 1$, associated with a van Hove-type peak in the density of states (this point will be discussed later). The corresponding crystal orbital has contributions from $\mathrm{Cu} 4 \mathrm{~s}, \mathrm{z}^{2}$ and $\mathrm{x}^{2}-\mathrm{y}^{2}$ with respective weights of $0.037,0.088$ and 0.252 . The $4 \mathrm{~s} / 3 \mathrm{z}^{2}$ hybridization is well known for the Jahn-Teller $\mathrm{Cu}^{2+}$ ion. The other contribution is from the $2 \mathrm{p}_{\mathrm{x}}$ orbital of one oxygen, with a weight of 0.650 . Thus, the oxygen character is approximately twice the copper character in this crystal orbital, and the bonding is strongly anisotropic with large antibonding interactions along [100] and nonbonding interactions along [010] (Figure 5e). This nonbonding character in X1 offers the possibility of opening a static or dynamical gap of nematic type $(a \neq b)$, with the creation of a double-well potential as in $\mathrm{SrTiO}_{3}$. For the Fermi level to be located in X1 for the $\sigma^{*}\left(\mathrm{~b}_{1 \mathrm{~g}}\right)$ band, a hole doping rate of $12-15 \%$ should be necessary. This approach, which agrees with experiment, does not discriminate electrons and doped holes; doping is used to shift the Fermi level toward a high-symmetry point of the Brillouin zone.

\subsection{The Double Unit Cell $\left(\mathrm{Cu}_{2} \mathrm{O}_{4}\right)^{4-}$}

Figure 5a shows the same dispersion curves $E(k)$ for a double unit cell $\left(\mathrm{Cu}_{2} \mathrm{O}_{4}\right)^{4-}$ but with a different crystal orbital distribution in the two unit cells. The hybridization of $z^{2}$ and $x^{2}-y^{2}$ reinforces the anisotropy of the (anti)bonding between copper and oxygen (Figure 5e). Besides, we can consider the hypothesis of a distribution of those two orbitals over two distinct sites (orbital ordering).

The correspondence between the Brillouin zones and high symmetry k-points of the single (BZ1, M1, X1 ... ) and double unit cell (BZ2, Г2, M2 .. ) is shown in Figure 4 (bottom).

The M2 point, corresponding to X1 for the single unit cell, shows a degeneracy between an energy band arising from $\sigma^{*}\left(\mathrm{~b}_{1 \mathrm{~g}}\right)(\mathrm{M} 1$ corresponds to $\Gamma 2)$ and a lower energy band arising from $2 e_{u}(\Gamma 1)$ (Figure 5a). Both bands show a full and opposite orbital ordering on copper sites labelled (1) and (2) (Figure 5d), and appeared isotropic since the weights on oxygen atoms were identical. The symmetries on the two copper sites were different: $a_{1 g}$ and $b_{1 g^{\prime}}$ which confirms the proposed 1:1 orbital ordering. Each oxygen atom, overall nonbonding, becomes a Janus entity, bonding on one side (with respect to 
$\mathrm{a}_{1 \mathrm{~g}} \mathrm{O}-\mathrm{O}$ bonding) and $\mathrm{Cu}-\mathrm{O}$ antibonding on both sides, but weakly in $\mathrm{a}_{1 \mathrm{~g}}\left(\mathrm{c}_{\mathrm{i}}^{2}=0.15\right)$, stronger in $\mathrm{b}_{1 \mathrm{~g}}$ $\left(c_{\mathrm{i}}^{2}=0.22\right)$, thus fully dissymmetric. Compared to X1 (spatially dissymmetric, antibonding/bonding along [100] and nonbonding along [010] (Figure 5e), with the reverse situation at Y1), M2 does not show any tendency to nematicity, but rather a strong tendency to couple to an optical mode of breathing type, with a dynamical inversion of the copper sites (1) and (2) (Peierls-type 2D instability); a tight-binding calculation (EHTB) leads to a gap opening of $33 \mathrm{meV}$ per pm of phonon amplitude.

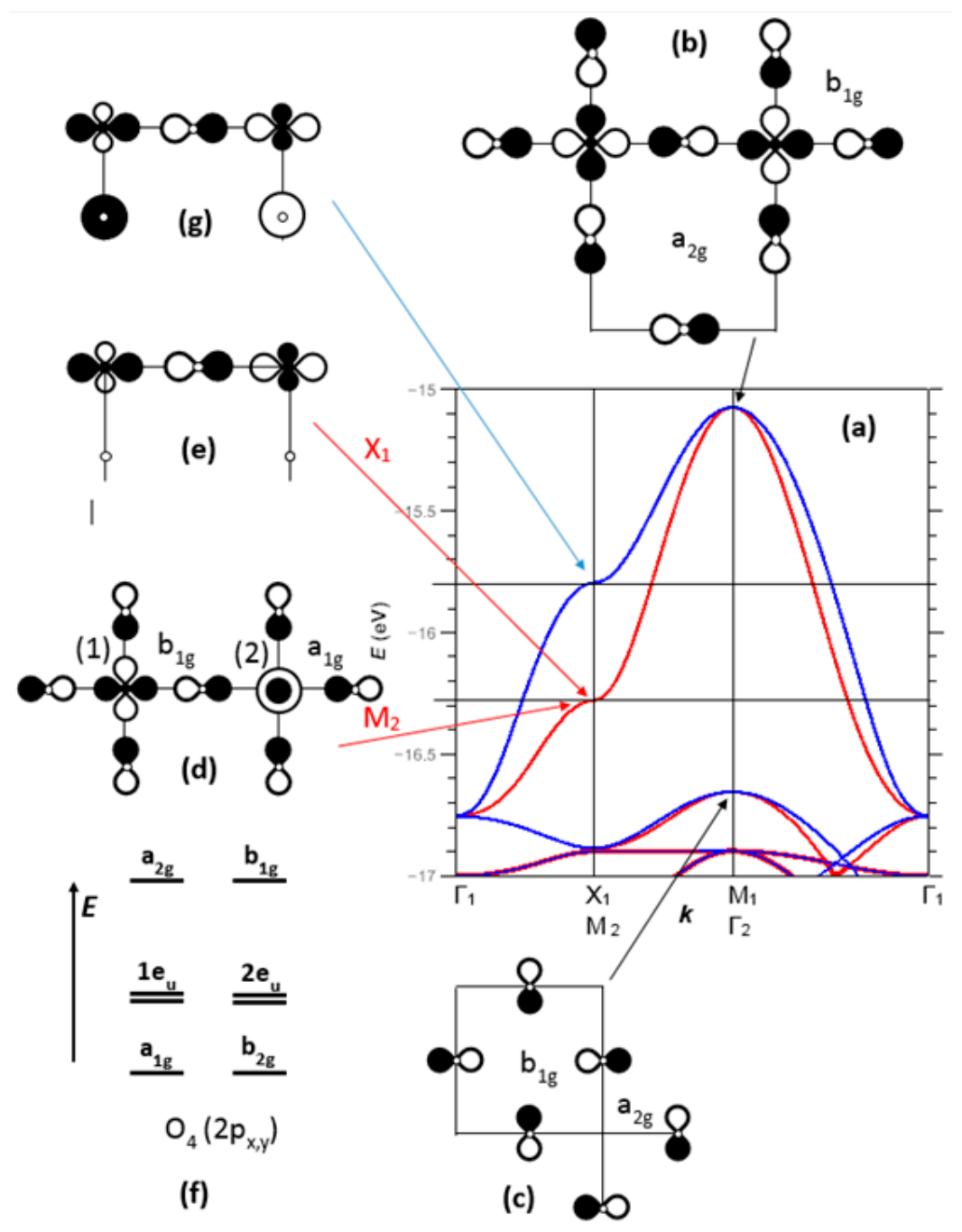

Figure 5. (a) Enlarged view of the two upper $\sigma^{*}\left(\mathrm{~b}_{1 \mathrm{~g}}\right)$ and $\pi^{*}\left(\mathrm{a}_{2 \mathrm{~g}}\right)$ bands of Figure 4 with (blue) and without (red) participation of oxygen 2s AOs. Representations of the crystal orbitals belonging to single and double unit cells (indexed 1 and 2, respectively): (b) $\sigma^{*}\left(\mathrm{~b}_{1 \mathrm{~g}}\right)$ crystal orbital in M1(Г2) with $\mathrm{b}_{1 \mathrm{~g}}$ and $\mathrm{a}_{2 \mathrm{~g}}$ symmetries for the copper occupied and non-occupied sites, respectively. (c) $\pi^{*}\left(\mathrm{a}_{2 \mathrm{~g}}\right)$ crystal orbital in M1, with $\mathrm{a}_{2 \mathrm{~g}}$ and $\mathrm{b}_{1 \mathrm{~g}}$ symmetries for the copper occupied and non-occupied sites, respectively. (d) Crystal orbital at the saddle point in M2, with two different copper sites of $b_{1 g}$ (1) and $\mathrm{a}_{1 \mathrm{~g}}$ (2) symmetry; in the middle, oxygen lies in a strongly asymmetric environment-a Janus atom-, with a large $2 \mathrm{p}-\left(\mathrm{x}^{2}-\mathrm{y}^{2}\right)$ overlap vs. a small $2 \mathrm{p}-\mathrm{z}^{2}$ overlap; $\mathrm{O}-\mathrm{O}$ interactions are antibonding around $\mathrm{b}_{1 \mathrm{~g}}$ and bonding around $\mathrm{a}_{1 \mathrm{~g}}$. (e) Crystal orbital with same anisotropy in X1 than (d), but not leading to 1:1 orbital ordering, by differentiating [100] from [010] directions. (f) Eight molecular orbitals for a square $\mathrm{O}_{4}$ group, considering $2 \mathrm{p}_{\mathrm{x}}$ and $2 \mathrm{p}_{\mathrm{y}}$ only. (g) Role of oxygen $2 \mathrm{~s}$ orbitals, decreasing the anisotropy found in (e).

We too had here a critical hypothesis, with an oscillating band gap driven by a phonon mode. At each half-period, the two bands cross; at this very moment, the noncrossing rule suggests the 
opening of an electronic gap, followed by a superconducting gap $2 \Delta_{\mathrm{SC}}$ by mixing the two bands according to:

$$
\left\langle\mathrm{e}_{\mathrm{u}}^{1}(\boldsymbol{k}) \cdot \overline{\mathrm{e}}_{\mathrm{u}}^{1}(-\boldsymbol{k})\left|H^{\prime}\right| \mathrm{b}_{1 \mathrm{~g}}^{0}\left(\boldsymbol{k}^{\prime}\right) \cdot \overline{\mathrm{b}}_{1 \mathrm{~g}}^{0}\left(-\boldsymbol{k}^{\prime}\right)\right\rangle
$$

Figure 6a shows the evolution of the ground state along a phonon half-period $(-\pi / 2$ to $+\pi / 2)$, from $a b_{1 g}\left(a_{1 g}\right)$ enlarged (compressed) site, as a function of the oxygen displacement $\delta$. Two types of domains can be observed: (i) on both sides, a smooth evolution of the $e_{u}$ and $b_{1}$ band edges with $\delta$; (ii) in the middle, a progressive mixing of $e_{u}$ and $b_{1}$, up to a full reversal. Figure $6 b$ represents the crystal orbitals before and after mixing for a 50:50 ratio.
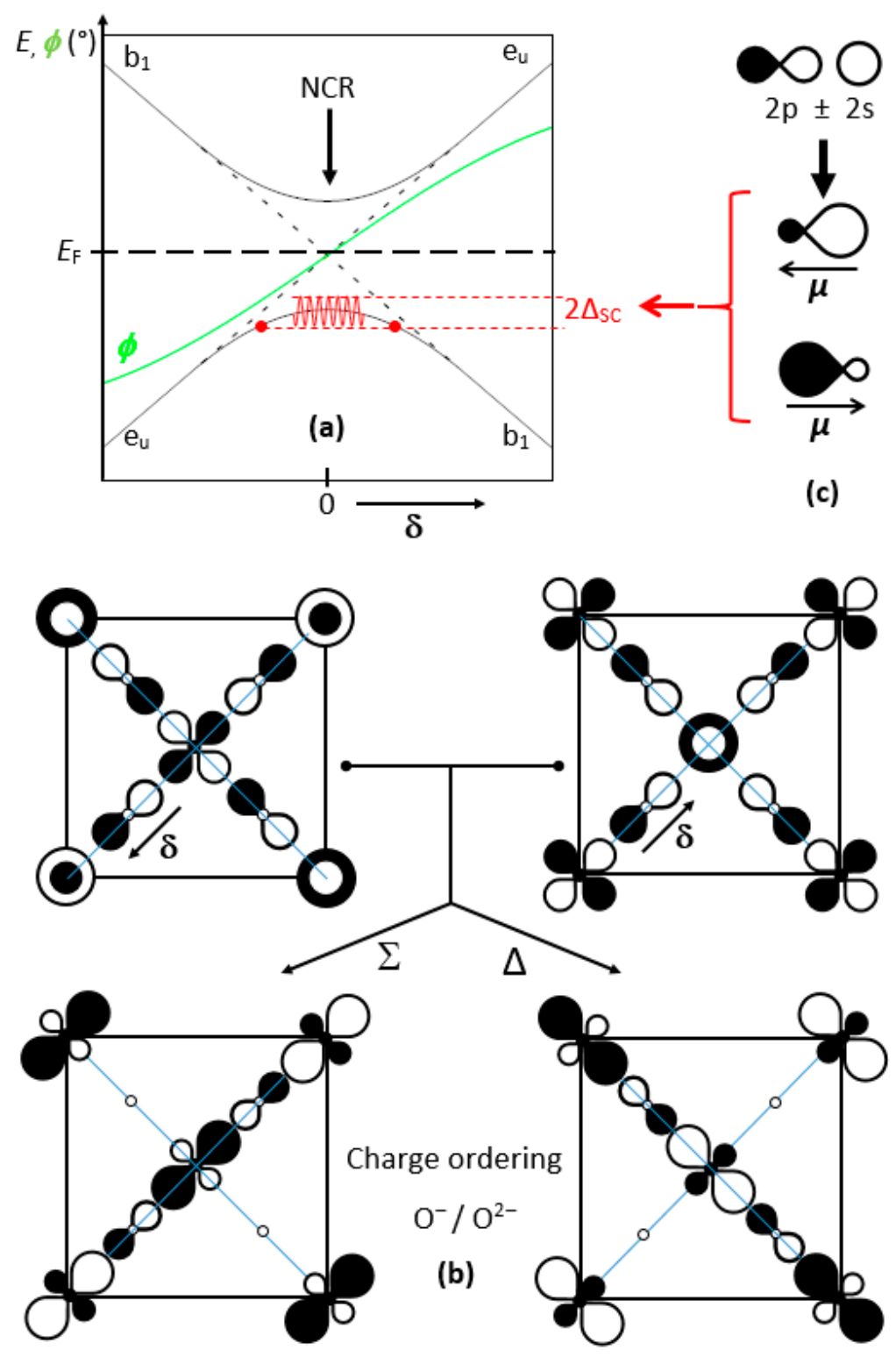

Figure 6. (a) Effect of the NCR along the phonon $\varphi$ of amplitude $\delta$. On the left side, the occupied $e_{u}$ band with large $b_{1 g}$ and small $a_{1 g}$ sites. On the right side, the same sites for the inverse $\delta$ and the occupied $b_{1}$ band. (b) Mixing the two bands at 50/50 and $\delta=0$ evens the copper charges and conversely differentiates strongly two among four oxygen atoms, inducing charge fluctuations. Hole doping creates $\left(\mathrm{O}_{4}\right)^{5-}$, which should disproportionate into $\left(\mathrm{O}_{4}\right)^{6-}$ and $\left(\mathrm{O}_{4}\right)^{4-}$. (c) Oxygen dipole reversal induced by the $2 \mathrm{~s}-2 \mathrm{p}$ hybridization and the phonon $\varphi$. 
The hybridization of $x^{2}-y^{2}$ and $z^{2}$ favors one direction out of [100] and [010], as observed above in $\mathrm{X} 1$, and evens the weights on both copper ions; conversely, it strongly discriminates the four oxygen atoms, two of them with vanishing crystal orbital coefficients. However, as we will discuss later, this charge ordering is likely to vanish partially by delocalization of the two holes among an $\left(\mathrm{O}_{4}\right)^{6-}$ cluster. From the above, it can be stated that such crystal orbital mixing leads to a charge transfer through electron (hole) pairs.

Another important feature is the high polarizability of $\mathrm{O}^{2-}$, whose electronic dipole (due to its Janus character) may oscillate at high frequency, a few orders of magnitude faster than phonons (red curve in Figure 6a). Such a behavior can be considered as the result of the $\mathrm{O}(2 \mathrm{p}) / \mathrm{O}(2 \mathrm{~s})$ hybridization (Figure 6c). This dipole oscillation may contribute, as in atomic spinodes, to induce a decomposition into various components of the $e_{u} / b_{1}$ mixtures in equilibrium, exchanging hole pairs, and consequently leading to the Whangbo Hamiltonian of pair-occupied and pair-unoccupied states [23,43].

\subsection{The $\pi^{*}\left(a_{2 g}\right)$ Band}

As proposed in 1990 by Micnas et al. and later illustrated by Simon [13,14], the crossing of a broad and a narrow band close to the Fermi level would be a key factor for the coupling of quasi-local pairs (narrow band) and fermions (broad band), to form Cooper pairs. Could the narrow, $300 \mathrm{meV}$-wide $\pi^{*}\left(\mathrm{a}_{2 \mathrm{~g}}\right)$ band, which lies approximately $400 \mathrm{meV}$ below the Fermi level, be raised to the Fermi level and generate local electron pairs? For this band, Figure 7 illustrates: (i) in X1(M2): the $a_{2 g}-b_{2 g}$ orbital ordering, with overall $\mathrm{O}-\mathrm{O}$ nonbonding character and $\mathrm{Cu}-\mathrm{O}$ antibonding character at the $b_{2 \mathrm{~g}}$ site; (ii) in M1: the $a_{2 g}$ symmetry of each copper site, for which oxygen atomic orbitals show $b_{1 g}$-type overlap on vacant cationic sites $\left(\frac{1}{2} \frac{1}{2} 0\right)$ with an antibonding $\mathrm{O}-\mathrm{O}$ character identical to the $\sigma^{*}\left(\mathrm{~b}_{1 \mathrm{~g}}\right)$ band for copper (see also Figure 5c).

\subsubsection{Positioning of the $\pi^{*}\left(\mathrm{a}_{2 \mathrm{~g}}\right)$ Band}

The $\left(\mathrm{CuO}_{2}\right)^{2-}$ layer is sandwiched between so-called reservoir layers, in which the doping adjusts the amount of charge carriers in the $\mathrm{CuO}_{2}$ layer. Additionally, being ionic, these reservoir layers generate an electrostatic (Madelung) potential that can be estimated by the Ewald summation method. One has to consider as well the screening of the Madelung potential by the charge carriers of the conducting layer; therefore, we restricted the calculation of Madelung potentials to the contribution of a few neighbor cells, and considered orders of magnitude only.

It is also straightforward to compare the importance of the $\mathrm{Cu}-\mathrm{O}$ charge transfer in $\mathrm{CuO}_{2}$ layers, by considering three cases of the Madelung terms: $\mathrm{Cu}^{2+} / \mathrm{O}^{2-}, \mathrm{Cu}^{+} / \mathrm{O}^{1.5-}$ and $\mathrm{Cu}^{0} / \mathrm{O}^{-}$. A few comments arise from these rough calculations: (i) as expected, the potential at the copper site is negative, whichever the $\mathrm{Cu}-\mathrm{O}$ charge transfer case is; (ii) the potential at the oxygen site is positive, except for the insignificant $\mathrm{Cu}^{0} / \mathrm{O}^{-}$case, and iii) at the vacant cationic site $\left(\frac{1}{2} \frac{1}{2} 0\right)$, which will be labeled $(+)$ in the following, the Madelung potential, $V_{\mathrm{M}}(+)$, is negative to whichever the charge transfer case is, but is lower by ca. $1 / 3$ than the potential at the occupied site, in absolute value. This justifies a specific treatment for the antibonding electrons in the $\pi^{*}\left(\mathrm{a}_{2 \mathrm{~g}}\right)$ band; iv) the negative character of $V_{\mathrm{M}}(+)$ increases when the apical $\mathrm{Cu}-\mathrm{O}$ distance (i.e., the separation between the $\mathrm{CuO}_{2}$ and the reservoir layer) increases. Therefore, the negative Madelung potential reinforces the destabilization of electrons at the vacant cationic site, adding up to the antibonding character of the $\mathrm{O}-\mathrm{O}$ interactions. These features should favor the stabilization of doped holes near the vacant $\left(\frac{1}{2} \frac{1}{2} 0\right)$ cationic sites of the $\mathrm{CuO}_{2}$ layer. How many holes? Can we envisage a hole pair on oxygen entities as well known for electrons and in $\mathrm{b}_{2 \mathrm{~g}}$ of electron doped $\mathrm{SrTiO}_{3}$, i.e., a dimerization $\left(\mathrm{O}_{4}\right)^{5-}+\left(\mathrm{O}_{4}\right)^{5-} \rightarrow\left(\mathrm{O}_{4}\right)^{4-}+\left(\mathrm{O}_{4}\right)^{6-}$ ? We would then obtain what chemists would call a hole lone pair, or an antiatom with a pseudo negative nucleus arising from $V_{\mathrm{M}}(+) \leq 0$; the two holes would then have a wave function of $\mathrm{x}^{2}-\mathrm{y}^{2}$ type of $b_{1 g}$ symmetry.

These distortions agreed perfectly with features revealed by Bianconi et al. [44] from extended X-ray absorption fine structure (EXAFS) spectroscopy on $\mathrm{La}_{1.85} \mathrm{Sr}_{0.15} \mathrm{CuO}_{4}$ crystals, showing a heterogeneous 
stripe system with an accumulation of hole carriers in a region with elongated apical and shortened equatorial Cu-O bonds ( $2.40 \AA$ and $1.88 \AA$, respectively).
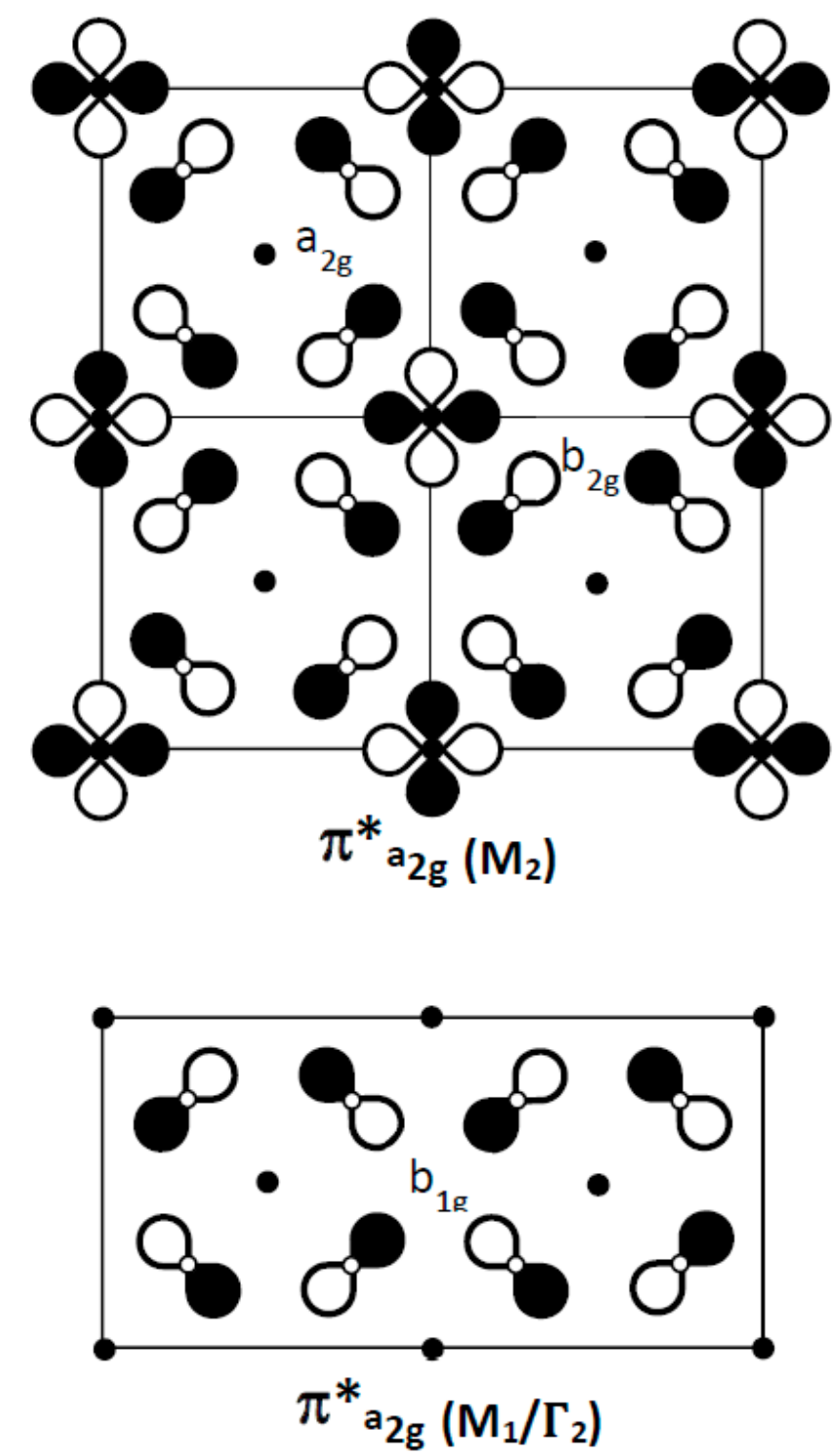

Figure 7. Crystal orbitals of the narrow $\pi^{*}\left(\mathrm{a}_{2 g}\right)$ bands at X1 (M2) and M1 points of the BZ.

\subsubsection{Mulliken-Jaffé Electronegativity for Oxygen}

In $M 1$, the $\pi^{*}\left(\mathrm{a}_{2 \mathrm{~g}}\right)$ band is of pure oxygen character. We can express the energy $E(q)$ of the oxygen ion as a function of its charge $q$, its electronic affinity $E A(q)$ and its ionization energy $E I(q)$ :

$$
E(q)=\alpha \cdot q+\beta \cdot q^{2}+\gamma \cdot q^{3}
$$

where $\alpha=\frac{1}{2} \cdot(E I(q)-E A(q))$ is the Mulliken electronegativity, $\beta$ is Pearson's hardness and $\gamma$ is an adjustable parameter; for oxygen, $\alpha=6.925, \beta=6.190$ and $\gamma=0.505$. $E(q)$ is plotted on Figure 8 . The Mulliken-Jaffé electronegativity $\chi_{\mathrm{MJ}}$ is defined as the derivative of $E(q)$ with respect to $q$. As seen in Figure $8, \chi_{\mathrm{MJ}}$ vanishes for $q \approx-0.6$ and is negative below this $q$ value. Additionally, it shows that $\mathrm{O}^{2-}$ is not stable in the gaseous phase, and will likely lose an electron at the surface of an oxide-catalysts take advantage of this effect.

Therefore, to account for the electronegativity of oxygen in an ionocovalent lattice, it is necessary to add a Madelung-type term in Equation (6). The magnitude of this term is difficult to estimate, 
since outer electrons of oxygen lie away from the exact atom site. Since the Madelung energy is proportional to $q^{2}$, we will use the corrected expression

$$
E(q)=\alpha \cdot q+\left(\beta-k^{\prime}\right) \cdot q^{2}+\gamma \cdot q^{3}
$$

for the energy of the oxygen ion as a function of its charge $q$. Figure 8 illustrates the importance of this Madelung term: (i) the minima are displaced toward the most negative charges (most ionic configurations), (ii) $E(q)$ vs. $q$ curves flatten, around their minima, until $k^{\prime} \approx 3$ and the appearance of an inflexion point. If we start from the $\left(\mathrm{O}_{4}\right)^{8-}$ cluster, oxidized into $\left(\mathrm{O}_{4}\right)^{6-}$ by charge transfer, at the X-point of the BZ, its progressive oxidation by doping into $\left(\mathrm{O}_{4}\right)^{5-}$ then $\left(\mathrm{O}_{4}\right)^{4-}$ corresponds to a maximum 0.5 electron per oxygen, for example between $\mathrm{O}^{-1}$ and $\mathrm{O}^{-1.5}$, whose formation energies $E(q=-1.0)$ and $E(q=-1.5)$ do not differ by more than $50 \mathrm{meV}$ (Figure 8 ). This energy difference is less than the $\mathrm{O}-\mathrm{O}$ resonance energy, which can be estimated to be approximately a hundred meV (Figure 4 of Ref. [36]); this leads to $U<0$, as in the negative- $U$ model for superconductivity [45].

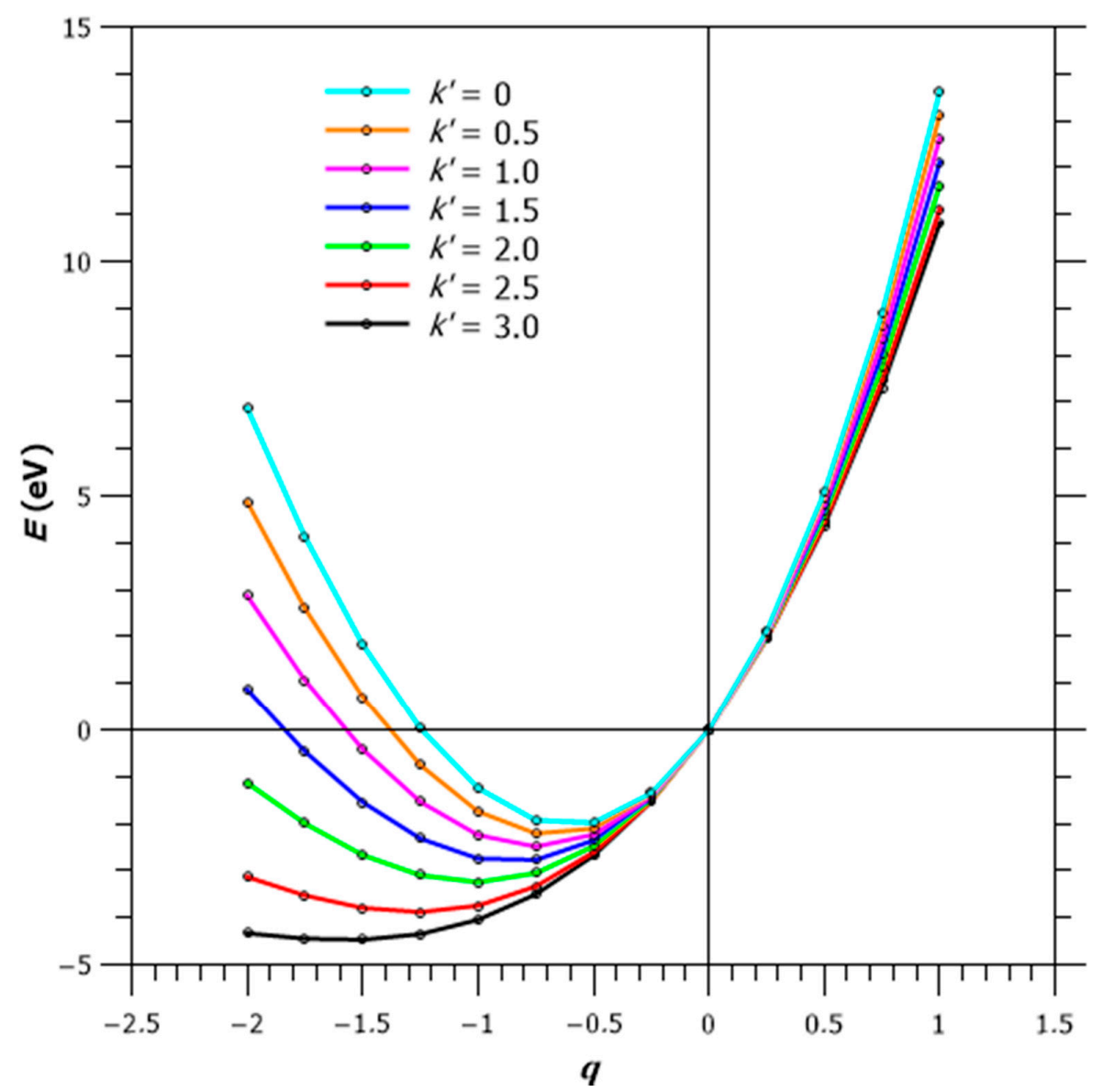

Figure 8. Formation energy $E(q)$ of an $\mathrm{O}^{q}$ ion $(-2<q<1)$, related to the Mulliken-Jaffe electronegativity $\chi_{\mathrm{M}-\mathrm{J}}$, for the free ion $\left(k^{\prime}=0\right)$ and for the ion embedded in a Madelung potential $\left(k^{\prime} \neq 0\right)$.

According to the above discussion, we can describe the electronic structure of $\left(\mathrm{CuO}_{2}\right)^{2-}$ layers by a network of $\mathrm{O}_{4}$ square planar entities (Figure 9), which provide the four sets of molecular orbitals $\left(b_{1 g}, a_{2 g}\right),\left(1 e_{u}, 2 e_{u}\right)$ and $\left(a_{1 g}, b_{2 g}\right)$ as indicated in Figure $5 f$ (by neglecting the out of plane $2 p_{z}$ orbitals), in order of decreasing energy. In this network, cationic sites are either (i) fully occupied by $\mathrm{Cu}^{2+}$ ions, in the tetragonal epitaxial form of $\mathrm{t}-\mathrm{CuO}$, (ii) half-occupied by $\mathrm{Cu}^{2+}$ in high- $T_{\mathrm{C}}$ cuprate layers, (iii) one-fourth occupied by $\mathrm{Cu}$ and one-fourth by $\mathrm{Li}^{+}$, in an ordered manner, in insulating $\mathrm{La}_{4} \mathrm{LiCuO}_{8}$, which can be described in terms of $\left(\mathrm{Li}^{+}\right)\left(\mathrm{Cu}^{+}\right)\left(\mathrm{O}_{4}\right)^{6-}$ layers $(\mathrm{I} / \mathrm{mmm})$, with two holes in the $\sigma^{*}\left(\mathrm{~b}_{1 \mathrm{~g}}\right)$ 
molecular orbital centered on each $\mathrm{Cu}^{+}$ion. At low $T$, this phase undergoes an orthorhombic distortion $(A m m m)$, from $\mathrm{D}_{4 \mathrm{~h}}$ to $\mathrm{D}_{2 \mathrm{~h}}$ for $\mathrm{Cu}^{+}$and $\mathrm{Li}^{+}$ions, with a buckling of distorted sites. Besides, the buckling of square-planar entities splits the unoccupied cationic sites (+) into two types of sites of the same $\mathrm{D}_{4}$ symmetry but a different size (labeled hereafter $\mathrm{L}$ and S). This atomic distortion and the increase in antibonding at the $S$ site are reinforced by a $2 p-2 s$ oxygen orbital hybridization towards the $S$ site (see below). These two situations can be transposed to the distortions of the $\mathrm{O}_{4}$ network of the $\left(\mathrm{Cu}_{2} \mathrm{O}_{4}\right)^{4-}$ layer, with a breathing mode (arrows in Figure 9) but also a "hole disproportionation" and octahedra buckling $\left(2 \mathrm{a}_{2 \mathrm{~g}}{ }^{1} \rightarrow \mathrm{a}_{2 \mathrm{~g}}{ }^{0}+\mathrm{a}_{2 \mathrm{~g}}{ }^{2}\right)$; this $\pi^{*}\left(\mathrm{a}_{2 \mathrm{~g}}\right)$ band, already narrow (ca. $\left.300 \mathrm{meV}\right)$ and further split by phonons, should serve as a seed for Cooper pairs delocalized in the broad $\sigma^{*}\left(2 \mathrm{e}_{\mathrm{u}}\right)$ band of the superconducting phase. This hole-acceptor character of $\pi^{*}\left(\mathrm{a}_{2 \mathrm{~g}}\right)$ agreed with the statement of Hirsh et al. for locating the cuprate holes preferentially in the oxygen $2 \mathrm{p}_{\pi}^{*}$ bands $[46,47]$.

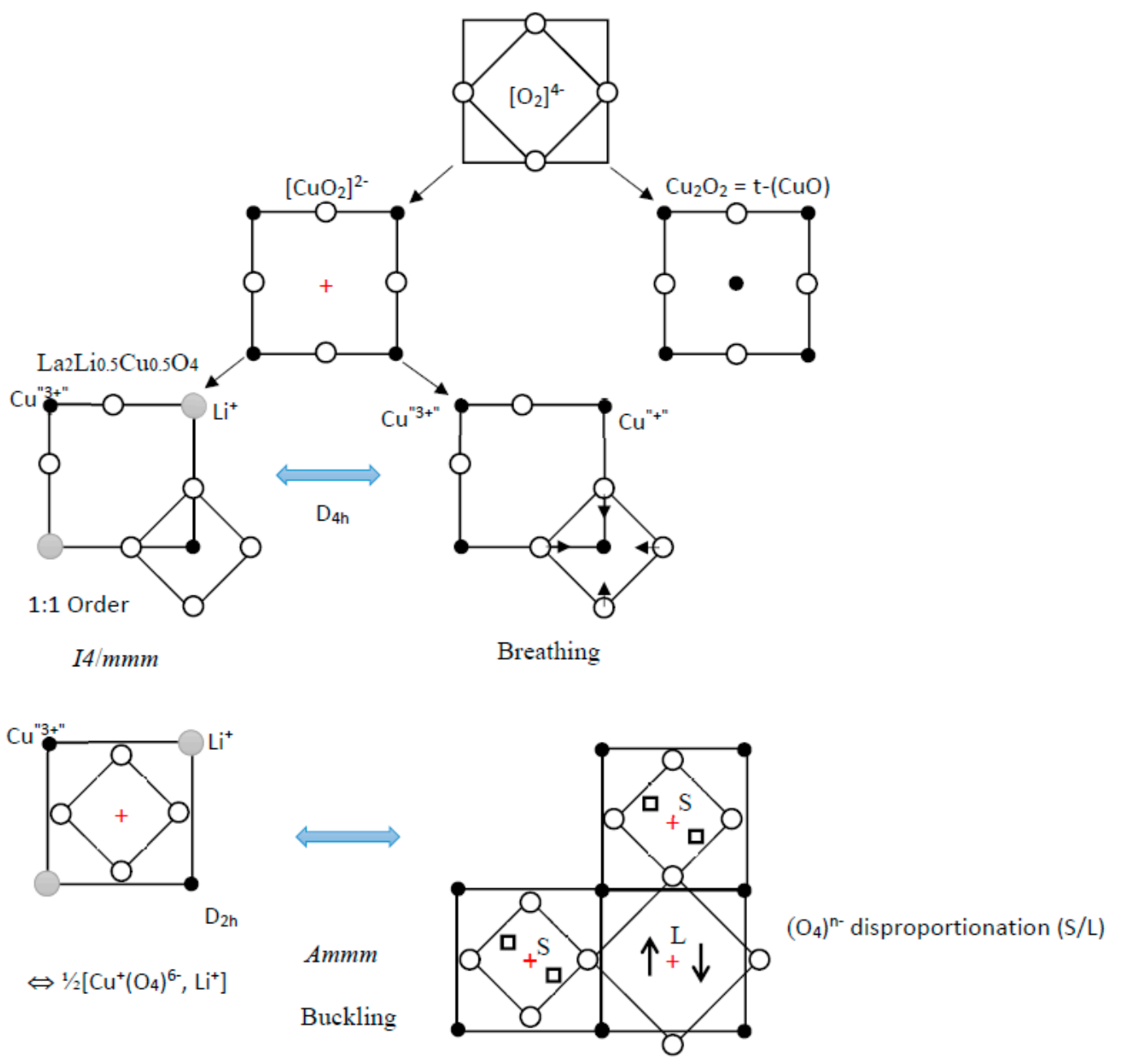

Figure 9. $\left[(\mathrm{Cu}, \mathrm{Li}) \mathrm{O}_{2}\right]^{2-}$ layers built from $\left(\mathrm{O}_{4}\right)^{\mathrm{n}-}$ square planar entities, with ordering or buckling, illustrating charge transfer as well as charge disproportionation possibilities. The small (S) and large (L) $\mathrm{O}_{4}$ squares represent such a case for $\left(\mathrm{O}_{4}\right)^{5-}$ groups.

\subsubsection{Role of Oxygen 2s Atomic Orbitals}

So far in our discussion, the role of semi-core (or rather deep-valence) $2 \mathrm{~s}$ orbitals of oxygen was not discussed; by introducing them in the tight-binding calculations, we noticed that the energy at $\mathrm{X} 1$ shifted upward by ca. $500 \mathrm{meV}$ (blue line in Figure 5a) due to $\mathrm{z}^{2}(\mathrm{Cu})-2 \mathrm{~s}(\mathrm{O})$ antibonding interactions along [010] (Figure 5g). Such a $2 s \pm 2 p$ hybridization modified the electron density in the vicinity of the oxygen ion, increasing the $\mathrm{Cu}-\mathrm{O}$ antibonding character of the band in an asymmetric way and contributing significantly to the Janus effect. In an axially distorted $\left(\mathrm{a}_{1 \mathrm{~g}}\right) \mathrm{Cu}_{2} \mathrm{O}_{4}$ 
cell, for example, the $2 s \pm 2 p$ hybridization reinforced the strong antibonding interactions with $x^{2}-y^{2}$, while it weakened the already weaker antibonding interactions with the equatorial lobe of $\mathrm{d}_{\mathrm{z}}{ }^{2}$ orbitals. Overall, this favored the charge transfer from oxygen to copper.

Similarly, for the Ammm transverse distortion, the $2 \mathrm{~s} \pm 2 \mathrm{p}$ hybridization reinforced the strong antibonding $\mathrm{O}-\mathrm{O}$ interactions in the small (S) square planar $\mathrm{O}_{4}$ groups, while weakening them in the large (L) ones. This favors the introduction of doping holes in this region of cationic type (negative value of the Madelung potential $V_{\mathrm{M}}$ ), even their pairing. Additionally, a much smaller hole density is necessary to reach the $\mathrm{X} 1$ point; it actually corresponds to a slight electron-doping (as in n-type cuprates). The contribution of $2 \mathrm{~s}$ orbitals will be modulated by the competing oxygen-rare earth bonding. The important role of oxygen $2 \mathrm{~s}$ orbitals had already been mentioned many years ago with respect to the gap value for the parent insulating phase [48].

\subsection{Underdoped Cuprates}

The phase diagram is complex (pseudo-gap, charge density waves, stripes, checkerboard patterns, etc. $[49,50])$; however, those phases should be related to characteristics of the pristine $\mathrm{La}_{2} \mathrm{CuO}_{4}$ phase. $\mathrm{La}_{2} \mathrm{CuO}_{4}$ is an insulating antiferromagnet of Mott or rather charge-transfer type, in which the lowest excitation involves the gap between the oxygen-character band and the upper Hubbard band: $\mathrm{O}^{2-}+\mathrm{Cu}^{2+} \rightarrow \mathrm{O}^{-}+\mathrm{Cu}^{+}$. This charge transfer can be symmetric, leading to an antiferromagnetic coupling by superexchange. We will examine the non-symmetric transfer only, which involves two copper ions (labeled 1 and 2) according to:

$$
\begin{gathered}
\uparrow \mathrm{Cu}(1)^{2+}+\mathrm{O}^{2-}+\downarrow \mathrm{Cu}(2)^{2+} \rightarrow \mathrm{Cu}(1)^{+}+\uparrow \mathrm{O}^{-}+\downarrow \mathrm{Cu}(2)^{2+}(\mathrm{U}), \text { or } \\
\uparrow \mathrm{Cu}(1)^{2+}+\mathrm{O}^{2-}+\downarrow \mathrm{Cu}(2)^{2+} \rightarrow \uparrow \mathrm{Cu}(1)^{2+}+\downarrow \mathrm{O}^{-}+\mathrm{Cu}(2)^{+}(\mathrm{D})
\end{gathered}
$$

where the arrows indicate the spin orientation. The remaining spin on oxygen couples with the spin $\frac{1}{2}$ on $\mathrm{Cu}(2)$, in a bonding state in Equation (8), in the same way as the Zhang and Rice singlet (ZRS) description [51] in the well-known $t-J$ model. The charge transfer (CT) of this type will be noted CT-ZRS(U) or CT-ZRS(D), by reference to the remaining spin of the oxygen ion. This transfer breaks the $\mathrm{C}_{2}$ symmetry of the $\mathrm{Cu}-\mathrm{O}-\mathrm{Cu}$ bridge, $\mathrm{Cu}^{+}$being larger than $\mathrm{Cu}^{2+}$ associated to $\mathrm{O}^{-}$. Thus, we can imagine a local two-dimensional 1:1 order of CT-ZRS(D) and CT-ZRS(U), which can be static or coupled to a phonon mode (see Figure 10). Three remarks arise:

(i) The charge transfer rate is formally $50 \%$ (i.e., half the optimal doping value),

(ii) One magnetic network over two must disappear at each phonon half-period; this scheme is consistent with the sharp decrease of Néel temperature with doping,

(iii) Local ordering domains appear ( $\mathrm{U}$ or $\mathrm{D})$, separated by antiphase boundaries, and alternating at each half-period.

Figure 10 (bottom) illustrates these two situations. What is the role of doping? At each half-period, when the symmetry is not broken, doping holes can form as many ZRS-[u] as ZRS-[d] (y-axis in Figure 10). However, the formation of CT-ZRS (either D or U) at each quarter-period, on each side of the antiphase boundary, is only compatible with doping holes of the opposite norm $(\mathrm{d} / \mathrm{U}$ or $\mathrm{u} / \mathrm{D})$, thereby rejecting doping holes of the same norm $(\mathrm{d} / \mathrm{D}$ or $\mathrm{u} / \mathrm{U})$ toward the other domain.

In essence, this is a collective displacement of holes of opposite spins ( $u$ vs. d) in opposite directions $(+\boldsymbol{k}$ vs. $-\boldsymbol{k})$, i.e., characteristic of Cooper pairs for a possible superconducting state. Note that this mechanism leads to a density of pairs, which is half the expected value, as seen experimentally [52].

In conclusion, this model agrees with the description by Reznick [53] titled "Electron-phonon coupling reflecting dynamic charge inhomogeneity in oxide superconductors", invoking strong bond stretching phonon anomalies common to stripe-ordered and superconducting materials, between ordered magnetic and hole-free layers, and charge transferred $\mathrm{Cu}^{+}$and hole-rich oxygen ones. 


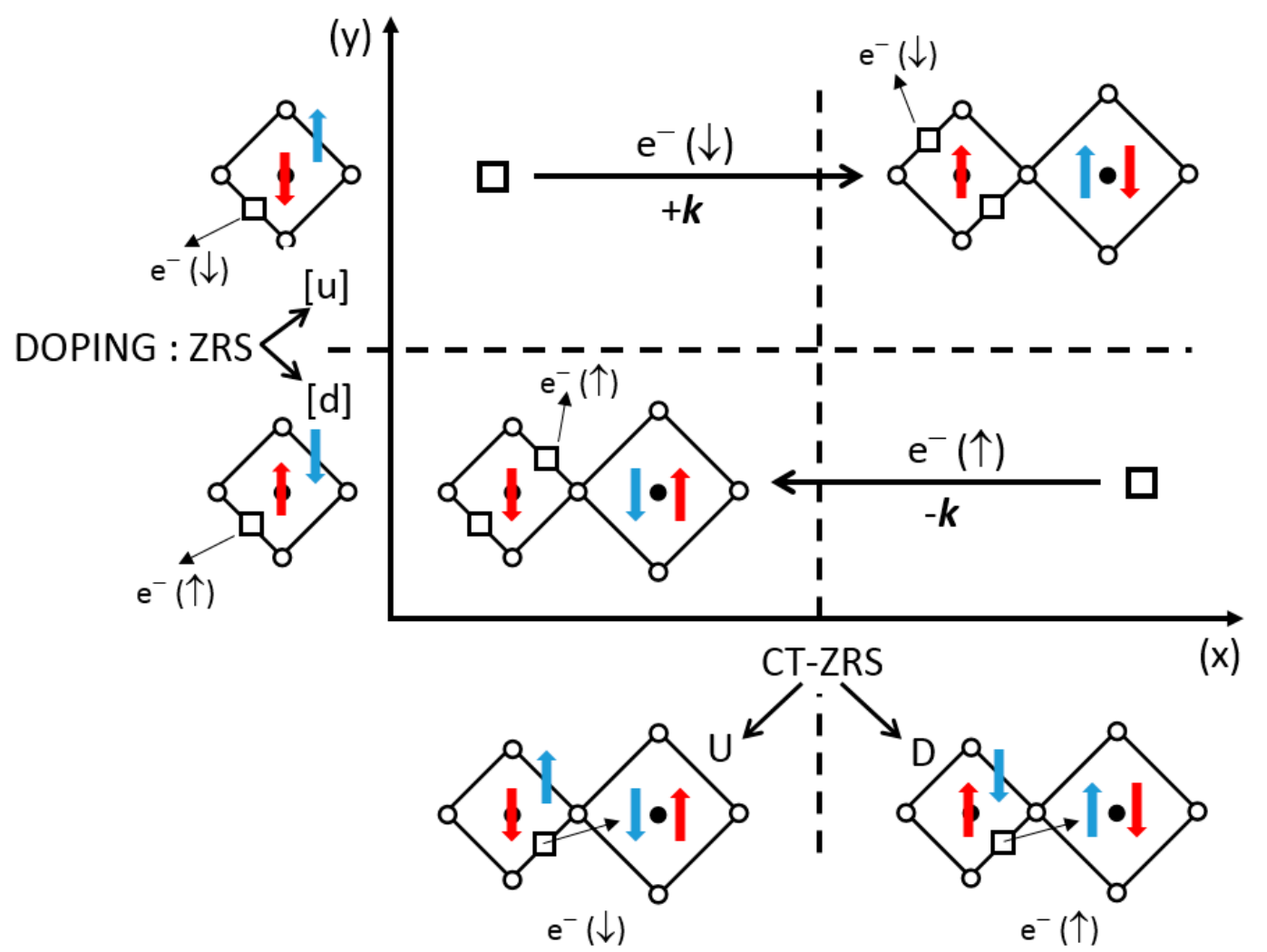

Figure 10. Phase diagram for a non-symmetric charge transfer (CT) in under-doped cuprates, as a function of hole doping (represented as usual by a Zhang-Rice singlet (ZRS) [51], and denoted as [u] or [d] according to the remaining oxygen spin) and CT between two copper atoms (denoted $U$ and $\mathrm{D}$ as previously). The diagram shows avoided areas, from where some doping holes ( $\mathrm{u} / \mathrm{U}$ or $\mathrm{d} / \mathrm{D})$ are expelled toward another CT-type part of the crystal, giving rise to hole transfer of opposite spin and momentum (Cooper pairs).

\section{Iron Arsenides and Selenides}

As early as 1965, Bertaut reported the anomalous nonmagnetic behavior of FeS, a rare mineral of mackinawite type, first identified in 1962 [54]. Due to its low thermal stability, FeS is difficult to obtain as a pure phase (i.e., without metallic iron as impurity). Besides, not long after the discovery of high- $T_{\mathrm{C}}$ superconductivity in the doped pnictide $\mathrm{LaO}_{1-x} \mathrm{~F}_{\mathrm{x}} \mathrm{FeAs}\left(T_{\mathrm{C}}=26 \mathrm{~K}\right)$ by Hosono et al. in 2008 [55], the selenide $\beta$-FeSe showed superconductivity below c.a. $10 \mathrm{~K}$. FeSe has the same mackinawite $(P 4 / \mathrm{nmm})$ structure as does FeS, but is more stable. Furthermore, $T_{\mathrm{C}}$ in FeSe can reach $30 \mathrm{~K}$ either under pressure or upon S-Se substitution; both conditions correspond to a decrease in the unit cell volume, as often observed for the spin transition $S=2 \rightarrow S=0$ in ferrous materials [56].

We chose to apply our tight-binding approach to FeSe, to check if similar features arise in the case of chalcogenides, as found in our analysis of the titanates and cuprates. The tight-binding parameters for Fe and Se are listed in Table 1. The values of Coulomb integrals for Fe 3d and Se 4p orbitals lie within $0.6 \mathrm{eV}$, indicating a strong covalency for Fe-Se bonds. This justifies the use of a vanishing $(U=0)$ Hubbard parameter for electron-electron repulsion, although a nonzero $U$ value would have to be considered for crystal orbitals of pure Fe character, at certain points of the Brillouin zone.

Figure 11 shows the crystal structure of $\beta$-FeSe. The iron atoms form a square plane network identical to the one found for the oxygen atoms in cuprates, an essential feature for our scenario of high- $T_{\mathrm{C}}$ superconductivity. However, the cationic vs. anionic character of Fe vs. O limits this analogy; in particular, the $E(q)$ curve for iron cannot show a minimum near $q=2$ (see Figure 8 for oxygen). Additionally, the cationic sites differ from an elongated octahedron $\left(\mathrm{D}_{4 \mathrm{~h}}\right)$ or a pyramid $\left(\mathrm{C}_{4 \mathrm{v}}\right)$ in cuprates to a slightly elongated tetrahedron $\left(T_{d}\right)$ in iron selenides or slightly compressed in iron 
pnictides. The distortion of the $\mathrm{FeSe}_{4}$ tetrahedron lifts the degeneracy of the iron $\mathrm{t}_{2}$ orbitals into an $e$ doublet (xz, yz) and a $b_{2}$ singlet (xy), only partly compensated by the crystal field $\left(\mathrm{D}_{4 \mathrm{~h}}\right)$ of the four $\mathrm{Fe}^{\mathrm{n}+}$ next-neighbors (at the distance of $2.67 \AA$ ).
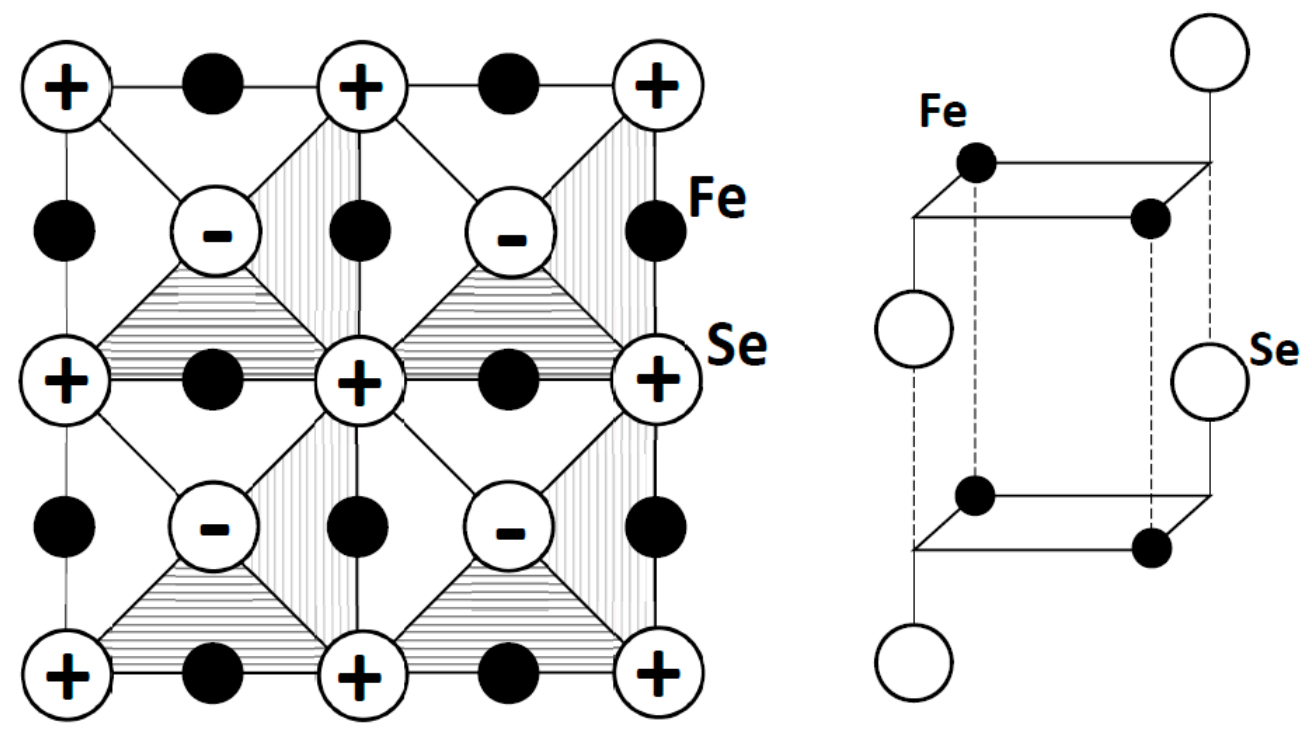

Figure 11. Scheme of the low-temperature structure of $\beta$-FeSe $(P 4 / n m m)$ with edge-sharing $\mathrm{FeSe}_{4}$ tetrahedra in the $a b$ plane (left), and the succession of layers along c (right).

Figure 12 shows the dispersion curves obtained from the tight-binding calculations for a $\mathrm{Fe}_{2} \mathrm{Se}_{2}$ primitive cell; the Fermi level is situated at $-11.74 \mathrm{eV}$. Several band stacks can be identified, in order of increasing energy: (i) around $-21 /-22 \mathrm{eV}$, two Se 4s-character bands (not represented in the figure); (ii) between ca. -15.5 and $-14 \mathrm{eV}$, six rather narrow Se $4 p$-character valence bands, with a significant Fe contribution and iii) broad conduction bands, divided in two stacks (according to the bonding or antibonding Fe-Fe interactions) that overlap slightly at $\Gamma 2$ and M2 points, close to the Fermi level. This creates two charge carrier pockets of $p$ type around $\Gamma 2$ and $n$ type around M2, with main components $x^{2}-y^{2}$ and $(x z, y z)$. We observe that the Fe-Fe bonding and antibonding bands of xy-character were well separated, lying respectively below and above the Fermi level, and corresponding to configurations $\sigma\left(b_{2}\right)^{2}$ and $\sigma^{*}\left(b_{2}\right)^{0}$.

The conduction band $\sigma^{*}(x z, y z)$ was broad (width $\left.>3 \mathrm{eV}\right)$; its top was strongly hybridized, with respective weights of $42 \%$ and $58 \%$ for $\mathrm{Fe}$ and Se, and its bottom was mostly of Fe character. It shows a progressive change into a broad domain (ca. $2 \mathrm{eV}$ ) with narrower bands built on $\mathrm{Fe}-\mathrm{Fe}$ interactions. It is close to half-filling, leading to a $(x z)^{1}(y z)^{1}$ configuration of Pauli-type states. The two $\mathrm{x}^{2}-\mathrm{y}^{2}$ bands, bonding and antibonding with respect to the Fe-Fe interactions, are noted as $1 \pi_{\mathrm{u}}$ and $1 \pi_{\mathrm{g}}$, respectively. Figure 13 represents a zoomed-in view of Figure 12 around M2 and close to $E_{F}$, for the six bands arising from $1 e_{u}, 2 e_{u}$ and $3 e_{u}$, based on $\left(x^{2}-y^{2} / 4 p_{x}, 4 p_{y}\right),(x z, y z)$ and $\left(x z, y z / 4 p_{z}\right)$ respectively. This corresponds to $a\left(x^{2}-y^{2}\right)^{1}$ configuration for Fe.

Two narrower $\mathrm{d}_{\mathrm{z}}^{2}$ bands are full, for a $\left(\mathrm{z}^{2}\right)^{2}$ configuration. Overall, this is consistent with divalent $\mathrm{Fe}$, with $\mathrm{xy}$ and $\mathrm{x}^{2}-\mathrm{y}^{2}$ electrons providing the strong cohesion of the network $\left(\mathrm{d}_{\mathrm{Fe}-\mathrm{Fe}}=2.67 \AA\right.$, close to the distance observed in $\gamma$-Fe). The hypothesis of a $\left(1 \pi_{\mathrm{u}}\right)^{2}\left(1 \pi_{\mathrm{g}}\right)^{0}$ configuration corresponds to a very low $U$ value. Otherwise, the spin polarization of these bands would induce a non-compensation magnetism, as observed in insulating phases of similar structure, with ordered Fe vacancies, such as $\mathrm{K}_{0.8} \mathrm{Fe}_{1.6} \mathrm{Se}_{2}\left(\mathrm{a} \sqrt{5} \times \mathrm{a} \sqrt{5}\right.$, S.G. I4/m) in which ferromagnetic $\mathrm{Fe}_{4}$ clusters order in an antiferromagnetic way below ca. $500 \mathrm{~K}$ [57]. 


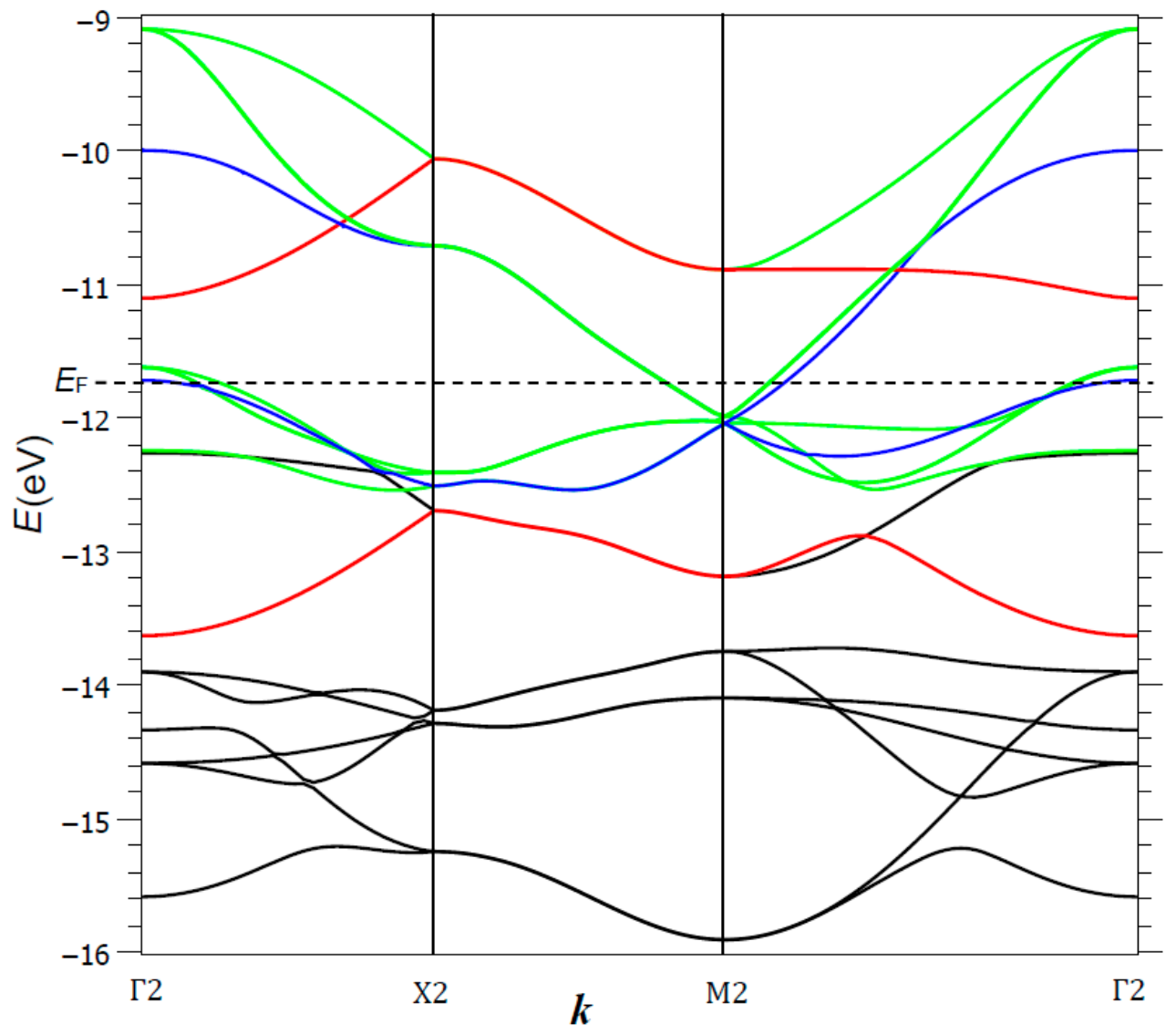

Figure 12. Dispersion curves $E(k)$ for the $\mathrm{Fe}_{2} \mathrm{Se}_{2}$ unit cell. Red: xy bands separated by a large gap between bonding and antibonding Fe-Fe interactions. Blue: $\left(x^{2}-y^{2}\right)$ bands, degenerate at the M2 point only. Green: conduction band based essentially on Fe (xz,yz) and Se 4p AOs.

The occurrence of $\mathrm{Fe}_{4}$ clusters recalls the $\mathrm{O}_{4}$ groups in the cuprates discussed above. Furthermore, the low temperature form of FeSe shows an orthorhombic distortion (S.G. Cmma, a/b = 0.9957) whose nematicity arises from the anisotropy of global Fe-Fe nonbonding interactions (bonding along $a$, antibonding along $b$ ) with a $a \sqrt{2}$ superstructure $[19,58]$. This led us to extend our tight-binding calculations to a double unit cell. The correspondences between crystal orbital notations in the single and double unit cells are given in Table 2. We compare in the following the crystal orbitals of such a double cell for the high temperature $\mathrm{P} 4 / \mathrm{nmm}$ and the low temperature Cmma forms, the latter presenting a nematic distortion; we suppose a phonon regime where the parameter ratio a/b oscillates around $1,(1 \pm \delta)$.

Table 2. Correspondence table for the symmetry of crystal orbitals in FeSe, from single $(Z=2)$ to double unit cell $(Z=4)$, and two space groups for the latter.

\begin{tabular}{ccc}
\hline $\mathrm{Z}=\mathbf{2}$ & $\mathrm{Z}=\mathbf{4}$ \\
\hline & $P 4 / n m m$ & $C m m a$ \\
\hline $1 \mathrm{e}_{\mathrm{u}}$ & $1 \pi_{\mathrm{g}}$ & $1 \sigma_{\mathrm{u}}$ \\
& $1 \pi_{\mathrm{u}}$ & $2 \sigma_{\mathrm{u}}$ \\
\hline $2 \mathrm{e}_{\mathrm{u}}$ & $2 \pi_{\mathrm{g}}$ & $1 \pi_{\mathrm{u}}$ \\
& $2 \pi_{\mathrm{u}}$ & $2 \pi_{\mathrm{u}}$ \\
\hline $3 \mathrm{e}_{\mathrm{u}}$ & $3 \pi_{\mathrm{g}}$ & $3 \pi_{\mathrm{u}}$ \\
& $3 \pi_{\mathrm{u}}$ & $4 \pi_{\mathrm{u}}$ \\
\hline
\end{tabular}




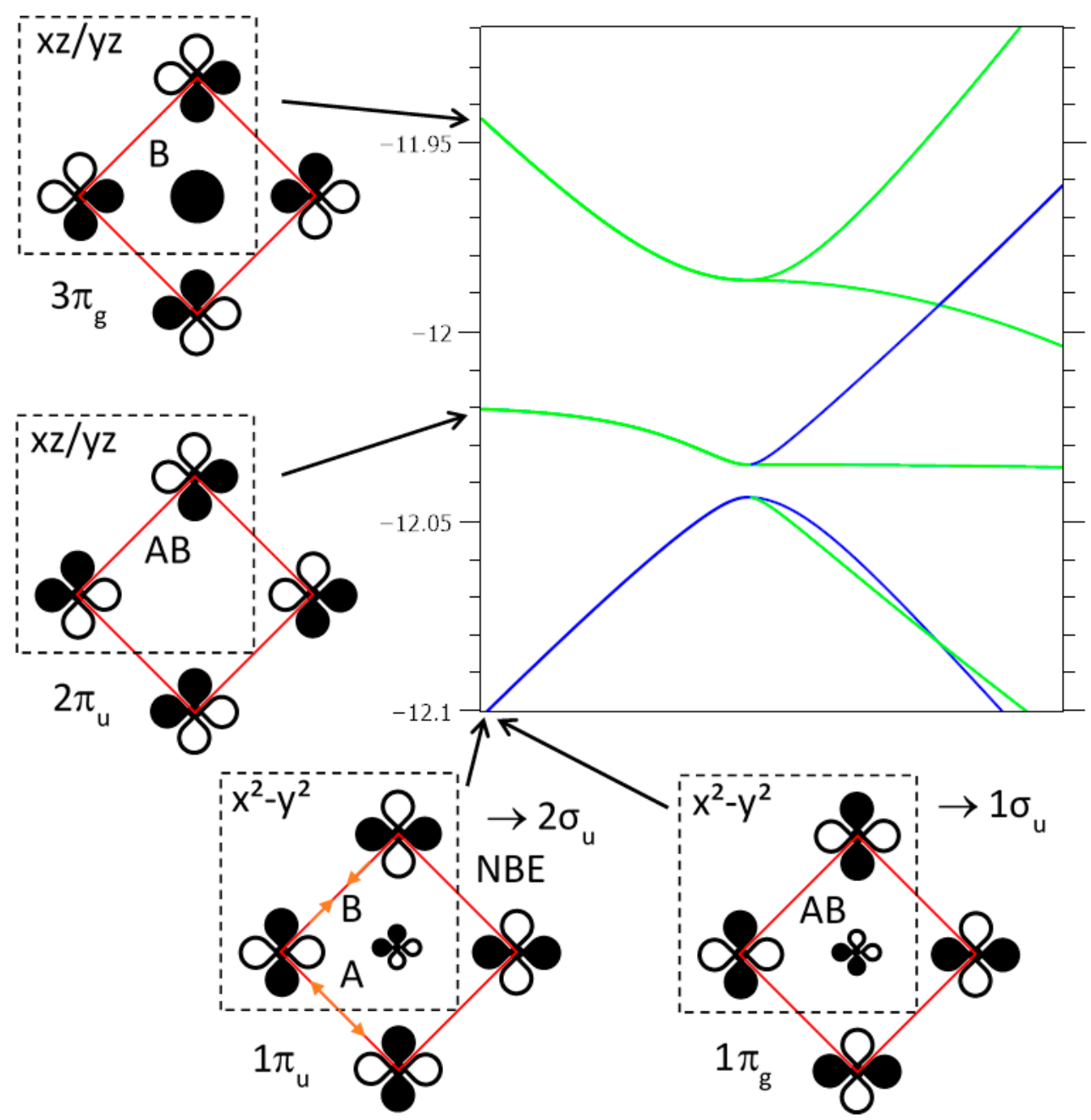

Figure 13. Enlargement of the dispersion curves of Figure 12, around $-12 \mathrm{eV}$, showing the three bands crossing at M2 ranked by increasing energy: $1 \pi_{\mathrm{g}} / 1 \pi_{\mathrm{u}}\left(1 \mathrm{e}_{\mathrm{u}}\right)$ in blue for $\mathrm{x}^{2}-\mathrm{y}^{2} ; 2 \pi_{\mathrm{g}}$ (not represented)/2 $\pi_{\mathrm{u}}$ $\left(3 e_{u}\right)$ in green for the $x z, y z$ purely Fe-Fe antibonding interactions, and $3 \pi_{\mathrm{g}} / 3 \pi_{\mathrm{u}}$ (not represented) $\left(3 e_{u}\right)$ mixed Se and Fe AOs (in green) with bonding Fe-Fe interactions in the broad conduction band. Note how $1 \pi_{\mathrm{g}}$ and $1 \pi_{\mathrm{u}}$ transform respectively in $1 \sigma_{\mathrm{u}}$ and $2 \sigma_{\mathrm{u}}$ for the double cell $\mathrm{Fe}_{4} \mathrm{Se}_{4}$.

We focus our attention on the three degenerate crystal orbitals in $M_{2}$, i.e., $1 e_{u}, 2 e_{u}$ and $3 e_{u}$ at respectively $-12.044,-12.035$ and $-11.986 \mathrm{eV}$, respectively, and their six split bands towards $\Gamma_{4}$ (note that, for the double cell, $\mathrm{x}^{2}-\mathrm{y}^{2}$ is transformed into $\mathrm{xy}$ ): (i) an antibonding-hybridized Fe $\mathrm{xy}$ with Se $4 p_{x}$ and $4 p_{y} A O s$ in an orbital ordering way, noted as $1 e_{u}\left(1 \pi_{g}, 1 \pi_{u} \rightarrow 1 \sigma_{u}, 2 \sigma_{u}\right)$. Globally, $\mathrm{Fe}-\mathrm{Fe}$ interactions are NB, the sum of bonding in the $a$ direction and antibonding in the $b$ direction, thus justifying the nematic instability. (ii) A purely $\mathrm{Fe}, 2 \mathrm{e}_{\mathrm{u}}\left(2 \pi_{\mathrm{g}}, 2 \pi_{\mathrm{u}} \rightarrow 1 \pi_{\mathrm{u}}, 2 \pi_{\mathrm{u}}\right)$ band involving equally distributed weights of $x z$ and $y z$ on each Fe atom, arranged in an antibonding manner with the first neighbors and antibonding/bonding ways with the second neighbors. (iii) An antibonding-hybridized $\mathrm{Fe}-\mathrm{Se}, 3 \mathrm{e}_{\mathrm{u}}\left(3 \pi_{\mathrm{g}}, 3 \pi_{\mathrm{u}} \rightarrow 3 \pi_{\mathrm{u}}, 4 \pi_{\mathrm{u}}\right)$ band, involving an orbital ordering, $\mathrm{xz}$ for the former and $\mathrm{yz}$ for the latter, mixed with Se $4 p_{z}$ AOs; each coefficient $c_{i}$ is close for each site. On the basis of degeneracies and regular weight distribution in the various sites, we cannot envisage any tendency of charge fluctuations, quoted here schematically "disproportionation", cationic and anionic, in the tetragonal phase.

What about the orthorhombic Cmma distortion? For a small iron atom displacement of $\pm 0.40 \mathrm{pm}$, the three bands in $\Gamma 4(=M 2)$ are split by 15,11 and $25 \mathrm{meV}$ respectively, allowing us to envisage a possible disproportionation. 
(i) For the pure iron $2 \pi_{\mathrm{u}} / 2 \pi_{\mathrm{g}}\left(2 \mathrm{e}_{\mathrm{u}}\right)$ bands, we observed an orbital ordering for yz and xz in the two bands; their mixing should only suppressed such an order.

(ii) For the Fe-Se hybridized $x y\left(x^{2}-y^{2}\right)$, i.e., the $1 \sigma_{u} / 2 \sigma_{u}$ bands, their coefficients $c_{i}$ were equally distributed on each atom ( \pm 0.46 for $\mathrm{Fe}, \pm 0.17$ for Se) conserving an orbital ordering. Distortion and iron oscillation away from their symmetrical positions (phonons), which favored bonding along $a$ (shortest Fe-Fe bonds) in $1 \sigma_{\mathrm{u}}$ and along $b$ in $2 \sigma_{\mathrm{u}}$, changed signs of some AO coefficients. As for the corresponding band of cuprates (Figure 6a), at each half period, the ground state changed from $1 \sigma_{\mathrm{u}}$ to $2 \sigma_{\mathrm{u}}$, violating the NCR of chemistry. To avoid that, the two bands did mix together, progressively up to a complete inversion, with three consequences:

- The creation of an electronic band gap,

- The different sign for the coefficients in the four iron sites between $1 \sigma_{\mathrm{u}}$ and $2 \sigma_{\mathrm{u}}$ bands inversed their sum $(\Sigma)$ or their difference $(\Delta)$,

- Passage during this mixture by an appropriate number of pair-occupied states and pair-unoccupied states may induce superconductivity with a $2 \Delta_{\text {SC }}$ gap $[23,43]$.

Using the previous values of $c_{1}$, we obtained for $\Sigma$ : $c_{1}=c_{2}=0 ; c_{3}=c_{4}= \pm 0.46$ for the lower 50:50 mixture (Figure 14), and for $\Delta: c_{1}=c_{2}= \pm 0.46$ and $c_{3}=c_{4}=0$ for the upper one. Two iron atoms out of four carry charge densities on their $x y\left(x^{2}-y^{2}\right)$ AOs corresponding to $4\left(\mathrm{x}^{2}-\mathrm{y}^{2}\right)^{1}=2\left(\mathrm{x}^{2}-\mathrm{y}^{2}\right)^{2}+2\left(\mathrm{x}^{2}-\mathrm{y}^{2}\right)^{0}$, or $4 \mathrm{Fe}^{2+}=2 \mathrm{Fe}^{+}+2 \mathrm{Fe}^{3+}$, qualitatively speaking. Such a succession along [100] and [010] of " $\mathrm{Fe}^{+}$" and " $\mathrm{Fe}^{3+}$ " allows for the Cooper pair formation and condensation at $T_{C}$ (Figure 15). Such a "disproportionation" is related to the Hubbard parameter $U$ for Fe $3 \mathrm{~d}$ orbitals and deals also with the difference between the third and the second ionization energies $(E I)$ of $\mathrm{Fe}$, which is the lowest $\left(E I_{3}-E I_{2}=14.5 \mathrm{~V}\right)$ value for the $\mathrm{M}^{2+} 3 \mathrm{~d}$ transition metal series, as shown in Table 3. However, a similar mixing between Se $4 p_{x}$ and $4 p_{y}$ AOs does not show any charge fluctuations in this $1 \sigma_{\mathrm{u}} / 2 \sigma_{\mathrm{u}}$ band.

Besides, the highly split $3 \mathrm{e}_{\mathrm{u}}\left(3 \pi_{\mathrm{u}} / 4 \pi_{\mathrm{u}}\right)$ states at the bottom of the wide conduction band with $\mathrm{xz} / \mathrm{yz}$ orbital ordering (Figure 13) differentiate two out of four selenium positions, above and below the iron layer: $\mathrm{c}_{1}(\Sigma)=-\mathrm{c}_{2}(\Sigma)=0.20 ; \mathrm{c}_{3}(\Sigma)=\mathrm{c}_{4}(\Sigma)=0$ and $\left.\mathrm{c}_{1}(\Delta)=\mathrm{c}_{2}(\Delta)=0 ; \mathrm{c}_{3}(\Delta)=-\mathrm{c}_{4}(\Delta)=0.20\right)$. Note that these states, upon mixing, do not induce Fe disproportionation.

The Se atoms form a square lattice of $\mathrm{a} / \sqrt{2}$ parameter (tetrahedron edge) above and below the iron layer with $\pi$-antibonding Se-Se interactions. At $\Gamma$ point, these interactions add to those of the Fe-Se antibonding character for destabilizing the top of the occupied states, favoring charge transfer. As discussed previously for the cuprates (Figure 8) for the $E(q)$ curve of oxygen, strong similarities were observed for selenium; the created holes can be coupled into pairs and the mixing of the $3 \pi_{\mathrm{u}} / 4 \pi_{\mathrm{u}}$ bands can also contribute to the superconducting gap $2 \Delta_{\mathrm{SC}}$ (Figure 15). Thus, the Fe-Se system disposed of two, cationic and anionic, phonon coupled carriers for coupling them into pairs and condensing them in Cooper pairs below $T_{C}$.

Similarly to the case of p-doped cuprates, we examined in which way the Fermi level could shift by a hundred meV to reach the $\mathrm{M}_{2}\left(\mathrm{x}^{2}-\mathrm{y}^{2}\right)$ point: this would be achieved through an electron transfer from $\left(x^{2}-y^{2}\right)^{*}$ to $(x z, y z)^{*}$ bands, i.e., from $\sigma$ to $\pi$, the latter being more stabilized by the intercalation of cations in the interlayer space, as demonstrated by Pickett and An for $\mathrm{MgB}_{2}$ vs. graphite [59]. The $30 \mathrm{~K}$ superconductors $\mathrm{A}_{0.6} \mathrm{Fe}_{2} \mathrm{Se}_{2}\left(\mathrm{~A}=\mathrm{M}^{+}\right)$illustrated such a behavior, increasing the amount of $\mathrm{Fe}^{+}$and consequently the Fermi energy, but conversely stabilizing $\pi^{*}(x z, y z)$ versus $\sigma^{*}\left(x^{2}-y^{2}\right)$ as discussed previously [60]. 


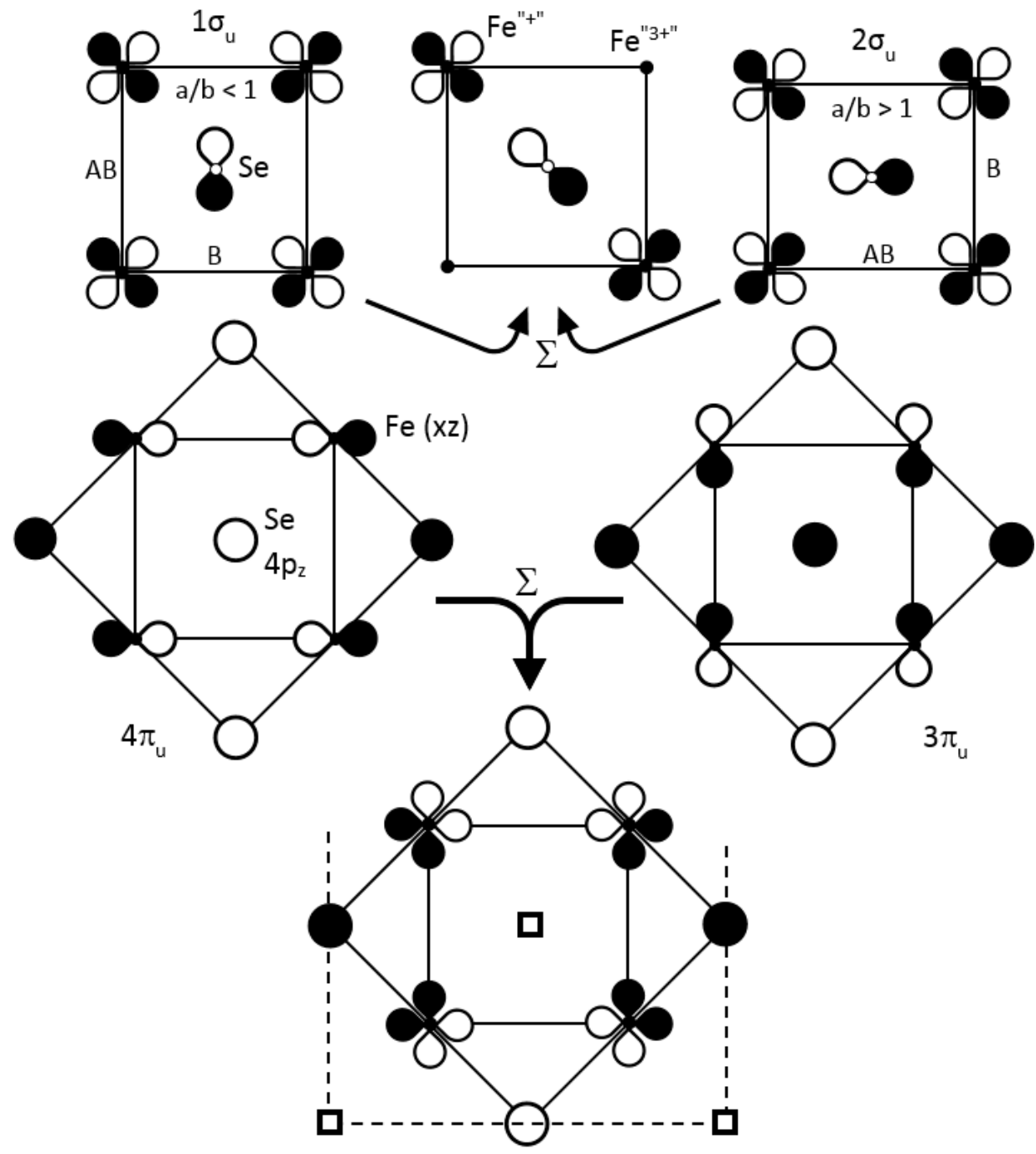

Figure 14. Orthorhombic Cmma distortion of the tetragonal $\mathrm{Fe}_{4} \mathrm{Se}_{4}$ double cell showing a nematic distortion along the corresponding phonon mode (see Figure 6$)$, for $1 \sigma_{u}(a / b<1$ and $B>A B$ on the left) and $2 \sigma_{\mathrm{u}}(\mathrm{a} / \mathrm{b}>1$ and $\mathrm{B}>\mathrm{AB}$ on the right), where $\mathrm{B}$ and $\mathrm{AB}$ represent the strengths of bonding and antibonding, respectively. $1 \sigma_{\mathrm{u}}$ and $2 \sigma_{\mathrm{u}}$ should mix during this inversion step, when the gap becomes smaller and smaller. The upper part shows the 50:50 mixing; from the AO coefficients in the two bands, two among four Fe atoms do not participate in the mixed wave function. This large charge fluctuation can be schematized as a phonon-induced "disproportionation" state implying electron-pair exchange. As shown in the lower part, the same distortion implies conversely, for $3 \pi_{\mathrm{u}} / 4 \pi_{\mathrm{u}}\left(3 \mathrm{e}_{\mathrm{u}}\right)$ mixing, a "disproportionation" state for Se atoms. Note that Se atoms lie above and below the Fe plane (see Figure 11); one layer out of two only contributes to the wave function.

Table 3. Values of 2nd and 3rd ionization energies of some transition metals, and their difference (in $\mathrm{eV}$ ).

\begin{tabular}{cccccccc}
\hline & Cr & Mn & Fe & Co & Ni & Cu & Zn \\
\hline$E I_{2}$ & 16.49 & 15.64 & 16.19 & 17.08 & 18.17 & 20.29 & 17.96 \\
$E I_{3}$ & 30.96 & 33.67 & 30.65 & 33.50 & 35.19 & 36.84 & 39.72 \\
$E I_{3}-E I_{2}$ & 14.47 & 18.03 & 14.46 & 16.42 & 17.02 & 16.55 & 21.76 \\
\hline
\end{tabular}



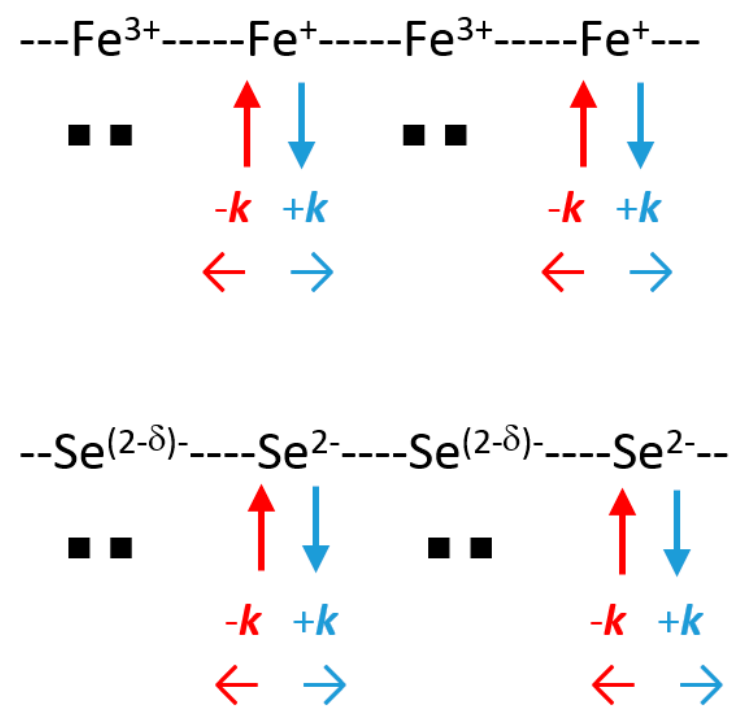

Figure 15. Top: Fe charge disproportionation in the Cmma unit cell of FeSe $(Z=4)$, by $1 \sigma_{\mathbf{u}}-2 \sigma_{\mathrm{u}}$ mixing along [100] and [010]. Bottom: charge fluctuation between successive (001) planes of selenium atoms, by $3 \pi_{\mathrm{u}}-4 \pi_{\mathrm{u}}$ mixing. Fe disproportionation and Se charge fluctuation are both consequences of the noncrossing rule.

\section{Methods}

Tight-binding calculations were done using the Extended Hückel method [61-64], as implemented in the CAESAR package [65], using parameters listed in Table 1.

\section{Conclusions}

The answer to the question in the title is clear: we believe that chemical bonding is at the heart of physical ordering phenomena and contributes to the understanding of the mechanisms of unconventional, high- $T_{C}$ superconductivity. It sheds light on the key role of nonbonding electrons, from which an electronic instability occurs through a double-well situation, inducing a static or dynamic (phonons) distortion of the atomic network. The coupling of nonbonding electrons with phonons was the core of our chemists' approach.

At low temperature, the softening of phonons led to a succession of symmetry broken and non-broken states, i.e., the alternation of an insulating distorted phase and a metallic phase. Importantly, it was also at the origin, on a shorter timescale, of a superconducting phase in which the mixing of occupied and unoccupied electron-pair states (described by Whangbo in $[23,43]$ ) could be achieved through a purely electronic Hamiltonian. This situation avoids the noncrossing rule for band-edge states, which should swap at each phonon half-period.

Besides, the approach of Micnas et al., based on the mixing of local pairs and itinerant electrons (when wide and narrow bands cross at the Fermi level) giving rise to a mutually induced superconductivity, was also relevant for our model. As discussed recently ([43] and references therein), such an interband electron pairing should also result from the breakdown of the Born-Oppenheimer approximation, favoring interband pair state excitations through nested Fermi surfaces.

The whole scheme applies to the three families of solids discussed here:

(i) Titanates, in which the top of the valence band was nonbonding and induced both a ferroelectric-type distortion, the splitting of the $\pi^{*}\left(\mathrm{t}_{2 \mathrm{~g}}\right)$ band into a narrow 2D band of $\mathrm{b}_{2 \mathrm{~g}}$ symmetry (xy) and a broader 2D band of eg symmetry (xz, yz),

(ii) Cuprates (at optimal p doping) had a wide conduction band, of oxygen character and $\sigma^{*}\left(\mathrm{~b}_{1 \mathrm{~g}}\right)$ symmetry, with a 2D Peierls-type instability for nonbonding electrons at the band center. 
This instability is probably of dynamical nature, and occurred at the Fermi level in the vicinity of a narrow band of pure oxygen character and of $\pi^{*}\left(\mathrm{a}_{2 \mathrm{~g}}\right)$ symmetry;

(iii) $\beta$-FeSe and iron pnictides of similar crystal structure had a broad conduction band (due to the strong Se (4p)-Fe (xz, yz) hybridization) and, close to the Fermi level, a much narrower band of Fe character, likely to split from a nonbonding situation into two bands of $x^{2}-y^{2}$ in character, which are $\mathrm{Fe}-\mathrm{Fe}$ bonding and antibonding, respectively.

In each family, the occurrence of bosonic local pairs was favored by specific features:

(i) A high dielectric constant that minimized the Coulomb repulsion between the two electrons of the $b_{2 g}{ }^{2}$ local pair $(U \rightarrow 0)$,

(ii) The occurrence of a poly-oxide (even peroxide) cluster $\left(\mathrm{O}_{4}\right)^{\mathrm{n}-}$, favored both by the almost constant formation energy of the $\mathrm{O}^{-q}$ ion for charges $q$ between -1 and -1.5 , a large transfer integral $t_{\mathrm{o}-\mathrm{o}}$ for hole delocalization, and the local crystal field at the copper-free(+) site, leading to a negative $U$ situation,

(iii) The existence of two $\left(x^{2}-y^{2}\right)$ sub-bands by splitting of $1 e_{u}$ in Cmma, of nonbonding $\mathrm{Fe}-\mathrm{Fe}$ character with Fe +II large charge fluctuations, crossing a broad 3D band of (xz,yz) character and strongly hybridized with the chalcogen. As in the case of oxygen atoms in the cuprates, the anionic Se layer underwent also similar charge fluctuations, likely coupled together.

These situations agreed perfectly with the requirements of the negative $U$ pairing mechanism, i.e., quasi degenerate states, same coordination and hybridization with the conduction band, as recently reported by Geballe, Hammond and Wu in a general introduction of superconductivity mechanisms [45].

The antagonism between several types of order can be discussed, magnetism vs. superconductivity, and magnetism vs. charge transfer: (i) as well known, magnetism destroys superconductivity above a critical field value, but the magnetic ordering is itself destroyed by charge transfer arising from p-doping in $\mathrm{Cu}+\mathrm{II}$ cuprates, leading eventually to a collapse of $T_{\mathrm{C}}$. However, both appear to be at the origin of superconductivity of under-doped cuprates, and their stripe-type insulating phases. (ii) Ferroelectricity and superconductivity seem to be incompatible, despite a few exceptions (see the discussion in Ref. [16]), because a significant carrier density is required (Mott transition). However, studies suggest that ferroelectric-type distortions also induce electron pairing. For both apparent antagonisms, the chemist' toolbox provides key arguments for possible interpretations.

Finally, the general model proposed here appeared as a coherent mixture of parts of physics and parts of chemistry: (i) the phonon role, alternating broken and unbroken symmetry, (ii) the broad and narrow band crossing of Micnas et al., (iii) the negative $U$ Hubbard model, as ingredients of the BCS theory, for physics, (iv) the non-bonding electron instability, (v) the non-crossing rule of states and bands and (vi) the charge-transfer disproportionation (which is equivalent to negative $U$ ), for chemistry. In addition, chemistry also suggests that anionic hybridization like $\mathrm{O}_{2 p} \pm \mathrm{O}_{2 \mathrm{~s}} \mathrm{AOs}$, rejecting alternatively electron densities on both sides from the symmetry center, describes very well the atomic polarizability and could be a high frequency alternative to phonons. In addition, coupling cationic and anionic disproportionation could enhance superconductivity as observed in cuprates and selenides.

Author Contributions: Conceptualization, mechanisms, elaboration, M.P.; validation, manuscript preparation, M.P. and A.V.; tight-binding calculations, manuscript finalization and editing, A.V. All authors have read and agreed to the published version of the manuscript.

Funding: This research received no external funding.

Conflicts of Interest: The authors declare no conflict of interest. 


\section{References}

1. Schafroth, M.R.; Blatt, J.M.; Butler, S.T. Quasichemical equilibrium approach to superconductivity. Helv. Phys. Acta 1957, 30, 93.

2. Little, W.A. Possibility of synthesizing an organic superconductor. Phys. Rev. A 1964, 134, 1416. [CrossRef]

3. Bardeen, J.; Cooper, L.; Schrieffer, J. Theory of superconductivity. Phys. Rev. 1957, 108, 1175. [CrossRef]

4. Pouchard, M.; Launay, J.C. Elaboration et caractérisation de monocristaux de $\mathrm{V}_{2} \mathrm{O}_{3}$ par transport en phase vapeur. Mater. Res. Bull. 1973, 8, 95. [CrossRef]

5. Chevrel, R.; Sergent, M.; Prigent, J. Sur de nouvelles phases sulfurées ternaires du molybdène. J. Solid State Chem. 1971, 3, 515. [CrossRef]

6. Matthias, B.T.; Marezio, M.; Corenzwit, E.; Cooper, A.S.; Barz, H.E. High-temperature superconductors, the first ternary system. Science 1972, 175, 1465. [CrossRef]

7. Goodenough, J.B.; Demazeau, G.; Pouchard, M.; Hagenmuller, P. Sur une nouvelle phase oxygénée du cuivre + III: SrLaCuO 4 . J. Solid State Chem. 1973, 8, 325. [CrossRef]

8. Bednorz, J.G.; Müller, K.A. Possible high-T $\mathrm{C}_{\mathrm{c}}$ superconductivity in the Ba-La-Cu-O system. Z. Phys. B Condens. Matter 1986, 64, 189. [CrossRef]

9. Nguyen, N.; Studer, F.; Raveau, B. Oxydes ternaires de cuivre à valence mixte de type $\mathrm{K}_{2} \mathrm{NiF}_{4}$ déficitaires en oxygène: évolution progressive d'un état semi-conducteur vers un état semi-métallique des oxydes $\mathrm{La}_{2-\mathrm{x}} \mathrm{Sr}_{\mathrm{x}} \mathrm{CuO}_{4-\mathrm{x} / 2+\delta}$. J. Phys. Chem. Solids 1983, 44, 389. [CrossRef]

10. Pouchard, M.; Grenier, J.C.; Doumerc, J.P. A simple cristallochemical model for the new superconducting copper oxides: A new class of mixed valencies with $\mathrm{N}$ and (N-2) electrons. C. R. Acad. Sci. 1987, 305, 571.

11. Simon, A. Superconductivity - a chemical phenomenon? Angew. Chem. 1987, 99, 602. [CrossRef]

12. Sleight, A.W. Rules of high-temperature superconductivity. Chemtronics 1987, 2, 116.

13. Micnas, R.; Ranninger, J.; Robaczkiewicz, S. Superconductivity in narrow-band systems with local nonretarded attractive interactions. Rev. Mod. Phys. 1990, 62, 113. [CrossRef]

14. Simon, A. Superconductivity and chemistry. Angew. Chem. Int. Ed. Engl. 1997, 36, 1788. [CrossRef]

15. Rischau, C.W.; Lin, X.; Grams, G.P.; Finck, D.; Harms, S.; Engelmayer, J.; Lorenz, T.; Gallais, Y.; Fauqué, B.; Hemberger, J.; et al. A ferroelectric quantum phase transition inside the superconducting dome of $\mathrm{Sr}_{1-\mathrm{x}} \mathrm{Ca}_{\mathrm{x}} \mathrm{TiO}_{3-\delta}$. Nat. Phys. 2017, 13, 643. [CrossRef]

16. Gabay, M.; Triscone, J.M. Ferroelectricity woos pairing. Nat. Phys. 2017, 13, 624. [CrossRef]

17. Pouchard, M.; Villesuzanne, A. A chemist's view of chemical bonding in the mechanism of high temperature superconductivity. arXiv 2017, arXiv:1707.00870.

18. Hosono, H.; Kuroki, K. Iron-based superconductors: Current status of materials and pairing mechanism. Physica C 2015, 514, 399. [CrossRef]

19. Chang, C.C.; Chen, T.K.; Lee, W.C.; Lin, P.H.; Wang, M.J.; Wen, Y.C.; Wu, P.M.; Wu, M.K. Superconductivity in Fe-chalcogenides. Physica C 2015, 514, 423. [CrossRef]

20. Wheeler, R.A.; Whangbo, M.H.; Hughbanks, T.; Hoffmann, R.; Burdett, J.K.; Albright, T. Symmetric vs. asymmetric linear M-X-M linkages in molecules, polymers, and extended networks. J. Am. Chem. Soc. 1986, 108, 2222. [CrossRef]

21. Deutscher, G.; de Gennes, P.G. A spatial interpretation of emerging superconductivity in lightly doped cuprates. C. R. Physique 2007, 8, 937. [CrossRef]

22. Orgel, L.E. Spectra of transition-metal complexes. J. Chem. Phys. 1955, 23, 1004. [CrossRef]

23. Whangbo, M.H. Electron Transfer in Biology and the Solid State; American Chemical Society: Washington, DC, USA, 1990.

24. Lin, X.; Bridoux, G.; Gourgout, A.; Seyfarth, G.; Krämer, S.; Nardone, M.; Fauqué, B.; Behnia, K. Critical doping for the onset of a two-band superconducting ground state in $\mathrm{SrTiO}_{3-\delta}$. Phys. Rev. Lett. 2014, 112, 207002. [CrossRef]

25. Migoni, R.; Bilz, H.; Bäuerle, D. Origin of raman scattering and ferroelectricity in oxidic perovskites. Phys. Rev. Lett. 1976, 37, 1155. [CrossRef]

26. Bussmann-Holder, A. The polarizability model for ferroelectricity in perovskite oxides. J. Phys. Cond. Matter 2012, 24, 273202. [CrossRef] [PubMed]

27. Bussmann-Holder, A.; Büttner, H.; Bishop, A.R. Polar-soft-mode-driven structural phase transition in $\mathrm{SrTiO}_{3}$. Phys. Rev. Lett. 2007, 99, 167603. [CrossRef] 
28. Szot, K.; Speier, W.; Bihlmayer, G.; Waser, R. Switching the electrical resistance of individual dislocations in single-crystalline $\mathrm{SrTiO}_{3}$. Nat. Mater. 2006, 5, 312. [CrossRef]

29. Muenstermann, R.; Dittmann, R.; Szot, K.; Mi, S.; Jia, C.L.; Meuffels, P.; Waser, R. Realization of regular arrays of nanoscale resistive switching blocks in thin films of $\mathrm{Nb}$-doped $\mathrm{SrTiO}_{3}$. Appl. Phys. Lett. 2008, 93, 023110. [CrossRef]

30. Bussmann-Holder, A.; Keller, H.; Simon, A.; Bihlmayer, G.; Roleder, K.; Szot, K. Unconventional co-existence of insulating nano-regions and conducting filaments in reduced $\mathrm{SrTiO}_{3}$ : Mode softening, local piezoelectricity, and metallicity. Crystals 2020, 10, 437. [CrossRef]

31. Hagenmuller, P. Non-Stoichiometric Compounds; Pergamon: Oxford, UK, 1975.

32. Edwards, P.P.; Sienko, M.J. Phase separation in metal solutions and expanded fluid metals. J. Am. Chem. Soc. 1981, 103, 2967. [CrossRef]

33. Gariglio, S.; Gabay, M.; Mannhart, J.; Triscone, J.M. Interface superconductivity. Physica C 2015, $514,189$. [CrossRef]

34. Clementi, E.; Roetti, C. Roothaan-Hartree-Fock atomic wavefunctions: Basis functions and their coefficients for ground and certain excited states of neutral and ionized atoms, $\mathrm{Z} \leq 54$. At. Data Nucl. Data Tables 1974, 14, 177. [CrossRef]

35. Demazeau, G.; Parent, C.; Pouchard, M.; Hagenmuller, P. Sur deux nouvelles phases oxygénées du cuivre trivalent: $\mathrm{LaCuO}_{3}$ et $\mathrm{La}_{2} \mathrm{Li}_{0.50} \mathrm{Cu}_{0.50} \mathrm{O}_{4}$. Mater. Res. Bull. 1972, 7, 913. [CrossRef]

36. Pouchard, M.; Doumerc, J.P.; Villesuzanne, A. Status of trivalent copper and charge-transfer excitons in high- $\mathrm{T}_{\mathrm{C}}$ cuprates. Inorg. Chem. 2008, 47, 8487. [CrossRef]

37. Bianconi, A.; Budnick, J.; Flanck, A.M.; Fontaine, A.; Lagarde, P.; Marcelli, A.; Tolentino, H.; Chamberland, B.; Michel, C.; Raveau, B.; et al. Evidence of $3 \mathrm{~d}^{9}$-ligand hole states in the superconductor $\mathrm{La}_{1.85} \mathrm{Sr}_{0.15} \mathrm{CuO}_{4}$ from $\mathrm{L}_{3}$ X-ray absorption spectroscopy. Phys. Letter A 1988, 127, 285. [CrossRef]

38. Mehta, A.; DiCarlo, J.; Navrotsky, A. Nature of hole states in cuprate superconductors. J. Solid State Chem. 1992, 101, 173. [CrossRef]

39. Hanaguri, T.; Lupien, C.; Kohsaka, Y.; Lee, D.H.; Azuma, M.; Takano, M.; Tagaki, H.; Davis, J.C. A 'checkerboard' electronic crystal state in lightly hole-doped $\mathrm{Ca}_{2-\mathrm{x}} \mathrm{NaxCuO}_{2} \mathrm{Cl}_{2}$. Nature 2004, 430, 1001. [CrossRef]

40. Koshaka, Y.; Taylor, C.; Fujita, K.; Schmidt, A.; Lupien, C.; Hanaguri, T.; Azuma, M.; Takano, M.; Eisaki, H.; Takagi, H.; et al. An intrinsic bond-centered electronic glass with unidirectional domains in underdoped cuprates. Science 2007, 315, 1380. [CrossRef]

41. Pasupathy, A.N.; Pushp, A.; Gomes, K.K.; Parker, C.V.; Wen, J.; Xu, Z.; Gu, G.; Ono, S.; Ando, Y.; Yazdani, A. Electronic origin of the inhomogeneous pairing interaction in the high- $\mathrm{T}_{\mathrm{C}}$ superconductor $\mathrm{Bi}_{2} \mathrm{Sr}_{2} \mathrm{CaCu}_{2} \mathrm{O}_{8+\delta}$. Science 2008, 320, 196-201. [CrossRef]

42. Jurkutat, M.; Kohlrautz, J.; Reichardt, S.; Erb, A.; Williams, G.V.M.; Haase, J. NMR of Cuprate Superconductors: Recent Developments. In High-T $T_{C}$ Copper Oxide Superconductors and Related Novel Materials; Bussmann-Holder, A., Keller, H., Bianconi, A., Eds.; Springer Series in Materials Science; Springer: Berlin/Heidelberg, Germany, 2017; Volume 255, pp. 77-87.

43. Whangbo, M.-H.; Deng, S.; Köhler, J.; Simon, A. Interband electron pairing for superconductivity from the breakdown of the Born-Oppenheimer approximation. ChemPhysChem 2018, 19, 3191. [CrossRef]

44. Bianconi, A.; Saini, N.L.; Lanzara, A.; Missori, M.; Rossetti, T.; Oyanagi, H.; Yamaguchi, H.; Oka, K.; Ito, T. Determination of the local lattice distortions in the $\mathrm{CuO}_{2}$ plane of $\mathrm{La}_{1.85} \mathrm{Sr}_{0.15} \mathrm{CuO}_{4}$. Phys. Rev. Lett. 1996, 76, 3412. [CrossRef] [PubMed]

45. Geballe, T.H.; Hammond, R.H.; Wu, P.W. What TC tells. Physica C 2015, 514, 9. [CrossRef]

46. Hirsch, J.E.; Tang, S. Hole superconductivity in oxides. Solid State Comm. 1989, 69, 987. [CrossRef]

47. Hirsch, J.E. Dynamic Hubbard model. Phys. Rev. Lett. 2001, 87, 206402. [CrossRef] [PubMed]

48. Villesuzanne, A.; Pouchard, M. New chemical bonding effects in narrow bands. Applications to to the superconducting transition temperature. C. R. Acad. Sci. Paris Ser. IIb 1996, 322, 155.

49. Bianconi, A. Superconductivity in quantum complex matter: The superstripes landscape. J. Supercond. Nov. Magn. 2020, 33, 2269. [CrossRef]

50. Innocenti, D.; Ricci, A.; Poccia, N.; Campi, G.; Fratini, M.; Bianconi, A. A model for liquid-striped liquid phase separation in liquids of anisotropic polarons. J. Supercond. Nov. Magn. 2009, 22, 529. [CrossRef] 
51. Zhang, F.C.; Rice, T.C. Effective Hamiltonian for the superconducting Cu oxides. Phys. Rev. B 1988, $37,3759$. [CrossRef]

52. Badoux, S.; Tabis, W.; Laliberté, F.; Grissonnanche, G.; Vignolle, B.; Vignolles, D.; Béard, J.; Bonn, D.A.; Hardy, W.N.; Lianget, R.; et al. Change of carrier density at the pseudogap critical point of a cuprate superconductor. Nature 2016, 531, 210. [CrossRef] [PubMed]

53. Reznick, D.; Pintschovius, L.; Ito, M.; Iikubo, S.; Sato, M.; Goka, H.; Fujita, M.; Yamada, K.; Gu, G.D.; Tranquada, J.M. Electron-phonon coupling reflecting dynamic charge inhomogeneity in copper-oxide superconductors. Nature 2006, 440, 1170. [CrossRef] [PubMed]

54. Bertaut, E.F.; Burlet, P.; Chappert, J. Sur l'absence d'ordre magnetique dans la forme quadratique de FeS. Solid State Comm. 1965, 3, 335. [CrossRef]

55. Kamihara, Y.; Watanabe, T.; Hirano, M.; Hosono, H. Iron-Based Layered Superconductor La[O1-xFx]FeAs $(\mathrm{x}=0.05-0.12)$ with $\mathrm{T}_{\mathrm{C}}=26 \mathrm{~K}$. J. Am. Chem. Soc. 2008, 130, 3296. [CrossRef] [PubMed]

56. Gütlich, P. Spin Cross-Over in Iron(II)-Complexes. In Structure and Bonding; Springer: Berlin/Heidelberg, Germany, 1981; p. 44.

57. Khasanov, R.; Bendele, M.; Conder, K.; Keller, H.; Pomjakushina, E.; Pomjakushin, V. Iron isotope effect on the superconducting transition temperature and the crystal structure of $\mathrm{FeSe}_{1-\mathrm{x}}$. New J. Phys. 2010, 12, 073024. [CrossRef]

58. Bao, W.; Huang, Q.Z.; Chen, G.-F.; Green, M.A.; Wang, D.-M.; He, J.-B.; Qiu, Y.-M. A novel large moment antiferromagnetic order in $\mathrm{K}_{0.8} \mathrm{Fe}_{1.6} \mathrm{Se}_{2}$ superconductor. Chin. Phys. Lett. 2011, 28, 086104. [CrossRef]

59. An, J.M.; Pickett, W.E. Superconductivity of $\mathrm{MgB}_{2}$ : Covalent bonds driven metallic. Phys. Rev. Lett. 2001, 86, 4366. [CrossRef]

60. Shoemaker, D.P.; Chung, D.Y.; Klaus, H.; Francisco, M.C.; Avci, S.; Llobet, A.; Kanatzidis, M.G. Phase relations in $\mathrm{K}_{\mathrm{x}} \mathrm{Fe}_{2-y} \mathrm{Se}_{2}$ and the structure of superconducting $\mathrm{K}_{\mathrm{x}} \mathrm{Fe}_{2} \mathrm{Se}_{2}$ via high-resolution synchrotron diffraction. Phys. Rev. B 2012, 86, 184511. [CrossRef]

61. Hoffmann, R. An extended Hückel theory. I. Hydrocarbons. J. Chem. Phys. 1963, 39, 1397. [CrossRef]

62. Whangbo, M.-H.; Hoffmann, R. The band structure of the tetracyanoplatinate chain. J. Am. Chem. Soc. 1978, 100, 6093. [CrossRef]

63. Ammeter, J.H.; Bürgi, H.B.; Thibeault, J.C.; Hoffmann, R. Counterintuitive orbital mixing in semiempirical and ab initio molecular orbital calculations. J. Am. Chem. Soc. 1978, 100, 3686. [CrossRef]

64. Wolfsberg, M.; Helmholz, L. The spectra and electronic structure of the tetrahedral ions $\mathrm{MnO}_{4}^{-}, \mathrm{CrO}_{4}^{-}$, and $\mathrm{ClO}_{4}^{-}$. J. Chem. Phys. 1952, 20, 837. [CrossRef]

65. Ren, J.; Liang, W.; Whangbo, M.-H. CAESAR Program Package. This Package Can Be Downloaded Free of Charge from the Website. 1998. Available online: http://www.primec.com/ (accessed on 23 October 2020).

Publisher's Note: MDPI stays neutral with regard to jurisdictional claims in published maps and institutional affiliations.

(C) 2020 by the authors. Licensee MDPI, Basel, Switzerland. This article is an open access article distributed under the terms and conditions of the Creative Commons Attribution (CC BY) license (http://creativecommons.org/licenses/by/4.0/). 